Grand Valley State University

ScholarWorks@GVSU

$1-27-2010$

\title{
Photodissociation of Ozone in the Hartley Band: Potential Energy Surfaces, Nonadiabatic Couplings, and Singlet/Triplet Branching Ratio
}

Reinhard Schinke

Max-Planck-Institut für Dynamik und Selbstorganisation, rschink@gwdg.de

George C. McBane

Grand Valley State University, mcbaneg@gvsu.edu

Follow this and additional works at: https://scholarworks.gvsu.edu/chm_articles

Part of the Biological and Chemical Physics Commons

\section{ScholarWorks Citation}

Schinke, Reinhard and McBane, George C., "Photodissociation of Ozone in the Hartley Band: Potential Energy Surfaces, Nonadiabatic Couplings, and Singlet/Triplet Branching Ratio" (2010). Peer Reviewed Articles. 19.

https://scholarworks.gvsu.edu/chm_articles/19

This Article is brought to you for free and open access by the Chemistry Department at ScholarWorks@GVSU. It has been accepted for inclusion in Peer Reviewed Articles by an authorized administrator of ScholarWorks@GVSU.

For more information, please contact scholarworks@gvsu.edu. 


\title{
Photodissociation of ozone in the Hartley band: Potential energy surfaces, nonadiabatic couplings, and singlet/triplet branching ratio
}

\author{
R. Schinke ${ }^{1, a)}$ and G. C. McBane ${ }^{2}$ \\ ${ }^{1}$ Max-Planck-Institut für Dynamik und Selbstorganisation, D-37073 Göttingen, Germany \\ ${ }^{2}$ Department of Chemistry, Grand Valley State University, Allendale, Michigan 49401, USA
}

(Received 2 December 2009; accepted 6 January 2010; published online 27 January 2010)

\begin{abstract}
The lowest five ${ }^{1} A^{\prime}$ states of ozone, involved in the photodissociation with UV light, are analyzed on the basis of multireference configuration interaction electronic structure calculations with emphasis on the various avoided crossings in different regions of coordinate space. Global diabatic potential energy surfaces are constructed for the lowest four states termed X, A, B, and R. In addition, the off-diagonal potentials that couple the initially excited state B with states $\mathrm{R}$ and A are constructed to reflect results from additional electronic structure calculations, including the calculation of nonadiabatic coupling matrix elements. The $\mathrm{A} / \mathrm{X}$ and $\mathrm{A} / \mathrm{R}$ couplings are also considered, although in a less ambitious manner. The photodissociation dynamics are studied by means of trajectory surface hopping (TSH) calculations with the branching ratio between the singlet, $\mathrm{O}\left({ }^{1} D\right)+\mathrm{O}_{2}\left({ }^{1} \Delta_{g}\right)$, and triplet, $\mathrm{O}\left({ }^{3} P\right)+\mathrm{O}_{2}\left({ }^{3} \Sigma_{g}^{-}\right)$, channels being the main focus. The semiclassical branching ratio agrees well with quantum mechanical results except for wavelengths close to the threshold of the singlet channel. The calculated $\mathrm{O}\left({ }^{1} D\right)$ quantum yield is approximately $0.90-0.95$ across the main part of the Hartley band, in good agreement with experimental data. TSH calculations including all four states show that transitions $\mathrm{B} \rightarrow \mathrm{A}$ are relatively unimportant and subsequent transitions $\mathrm{A} \rightarrow \mathrm{X} / \mathrm{R}$ to the triplet channel are negligible. (C) 2010 American Institute of Physics. [doi:10.1063/1.3299249]
\end{abstract}

\section{INTRODUCTION}

The photodissociation of ozone in the near ultraviolet, an essential process in the atmosphere, ${ }^{1}$ has been studied in great detail. ${ }^{2,3}$ In this wavelength region the absorption cross section has a broad maximum at about $256 \mathrm{~nm}$ - the Hartley band-with very diffuse structures superimposed. ${ }^{4}$ Because of its importance for atmospheric chemistry, the dissociation dynamics of ozone in the Hartley band has been the subject of many theoretical studies; ${ }^{5-9}$ for a more complete list of references see the review article by Grebenshchikov et al. ${ }^{10}$

The Hartley band is due to excitation of the third state with ${ }^{1} A^{\prime}$ symmetry $\left({ }^{1} B_{2}\right.$ in $\left.C_{2 v}\right)$ :

$$
\begin{aligned}
\mathrm{O}_{3}\left(X^{1} A^{\prime}\right)+h \nu & \rightarrow \mathrm{O}_{3}\left({ }^{1} A^{\prime}\right) \\
& \rightarrow \mathrm{O}\left({ }^{3} P\right)+\mathrm{O}_{2}\left(X^{3} \Sigma_{g}^{-}\right) \quad(8 \%), \\
& \rightarrow \mathrm{O}\left({ }^{1} D\right)+\mathrm{O}_{2}\left(a^{1} \Delta_{g}\right) \quad(92 \%) .
\end{aligned}
$$

The main dissociation channel is the "singlet channel" Eq. (2), in which $\mathrm{O}$ and $\mathrm{O}_{2}$ are electronically excited; it diabatically correlates with $\mathrm{O}_{3}\left(3^{1} A^{\prime}\right)$. The significant population of the "triplet channel" Eq. (1), ${ }^{2,3}$ with both $\mathrm{O}$ and $\mathrm{O}_{2}$ in their ground electronic states, indicates, however, that the dissociation proceeds on more than one potential energy surface (PES). The electronic structure calculations show that in addition to $3{ }^{1} A^{\prime}$ the second state with ${ }^{1} A^{\prime}$ symmetry $\left({ }^{1} A_{1}\right.$ in $C_{2 v}$ ) and another state of the same symmetry, which is strongly repulsive, must also be considered. ${ }^{8,11,12}$ Following

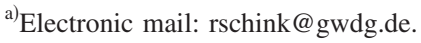

Hay et al. ${ }^{13}$ these three (diabatic) states will be termed B, A, and R, respectively. Both B and A correlate with channel (2) while R correlates with channel (1). Transitions to and dissociation via the $\mathrm{X}$ state are in principle also possible. With increasing photon energy the dissociating molecule will access regions of coordinate space where even more excited states are required for understanding the dynamics. For wavelengths shorter than $234.1 \mathrm{~nm}$ the excited triplet channels $\mathrm{O}\left({ }^{3} P\right)+\mathrm{O}_{2}\left({ }^{3} \Delta_{u}\right)$ and $\mathrm{O}\left({ }^{3} P\right)+\mathrm{O}_{2}\left({ }^{3} \Sigma_{u}^{+}\right)$, for example, are open and according to a recent experimental investigation are indeed populated, albeit with very small quantum yield of less than $1 \%{ }^{14}$ Accompanying electronic structure calculations, including up to ten ${ }^{1} A^{\prime}$ states, elucidated the pathways leading to the excitation of these so-called Herzberg states of $\mathrm{O}_{2}$.

Three-dimensional diabatic PESs for electronic states X, $\mathrm{A}, \mathrm{B}$, and $\mathrm{R}$, were determined by $\mathrm{Qu}$ et al. ${ }^{9}$ employing a simple diabatization "by eye." Adiabatic energies that apparently belonged to the same diabatic state were collected and the corresponding smooth PESs were fitted to analytical potential expressions. All B-state PESs published up to the present and used in dynamics calculations have been constructed in this way. ${ }^{8,9,13,15}$ Although diabatization by eye is a very crude procedure, the resulting PESs provide an overview of the crossing seams and therefore are helpful in understanding the possible fragmentation paths. However, the off-diagonal potential coupling elements, which govern the transitions among the diabatic states, cannot be determined by such a simple approach.

Although theoretical studies of the dissociation in the 
Hartley band have been ample, ${ }^{10}$ there are still several open questions. None of the calculations performed up to nowwith one exception, ${ }^{10}$ all using only the diabatic B-state PES and total angular momentum $J=0$ or $1-$ gave a satisfactory absorption spectrum; the vibrational structures superimposed on the broad absorption maximum were considerably too intense in comparison with the experimental spectrum..$^{5,6,9,16-19}$ But even the single study that included thermal broadening $(0 \leq J \leq 50)$ and predissociation due to coupling to the repulsive R-state did not yield conclusive agreement. ${ }^{10}$ The quantum yield in the triplet channel, $\Gamma\left({ }^{3} P\right)$, is mainly controlled by the coupling $V_{\mathrm{BR}}$ between states $\mathrm{B}$ and $\mathrm{R}$, which has not been adequately determined yet. $\mathrm{Qu}$ et $a l{ }^{20}$ introduced an effective parameter in order to adjust $\Gamma\left({ }^{3} P\right)$ in their semiclassical study; the internal energy distribution of $\mathrm{O}_{2}\left({ }^{3} \Sigma_{g}^{-}\right)$and its relation to the coordinate dependence of $V_{\mathrm{BR}}$ were the main focus. Other couplings, those between $\mathrm{B}$ and $\mathrm{A}$ or $\mathrm{A}$ and $\mathrm{R}$, for example, have not been discussed at all up to now.

In the present article we extend previous studies of the dissociation in the Hartley band. ${ }^{9}$ We describe new electronic structure calculations including the five lowest ${ }^{1} A^{\prime}$ states; they are more accurate and more complete than those previously published. On the basis of these calculations we determine new diabatic PESs for states X, B, A, and R. Furthermore, we determine the coupling elements between the diabatic states, especially the coupling between B and R. The fragmentation mechanisms and the branching ratios into the singlet and triplet channels, $\Gamma\left({ }^{1} D\right)$ and $\Gamma\left({ }^{3} P\right)$, are investigated by means of trajectory surface hopping (TSH) calculations and the results are compared with experimental data.

\section{ELECTRONIC STRUCTURE CALCULATIONS AND OVERVIEW OF POTENTIAL ENERGY SURFACES}

The electronic structure calculations are similar to those described by Qu et al., ${ }^{11,12}$ Zhu et al., ${ }^{21}$ and Baloïtcha and Balint-Kurti. ${ }^{8}$ The internally contracted multireference configuration interaction method with single and double excitations (MRD-CI) is employed. ${ }^{22,23}$ The CI wave functions are based on state-averaged complete active space self-consistent field (CASSCF) orbitals with 18 electrons in 12 orbitals (full-valence active space) and three fully optimized closedshell inner orbitals. The averaging includes the five lowest ${ }^{1} A^{\prime}$ states with equal weights. The subsequent CI calculations are carried out with 18 correlated valence electrons and 6 frozen-core electrons. In contrast with earlier calculations, ${ }^{11,12,21}$ all configurations are included, i.e., no selection of the most important configurations according to a cutoff threshold is applied. The Davidson correction is used to approximately account for contributions of higher excitations and for size-extensive energies. ${ }^{24}$ Dunning's standard augmented correlation consistent triple-zeta (aug-cc-pVTZ) basis set is used throughout. ${ }^{25}$ Energies are calculated for the five lowest ${ }^{1} A^{\prime}$ states. All calculations are carried out with the MOLPRO suite of programs. ${ }^{26}$

In what follows, $R_{1}$ and $R_{2}$ are the distances between the central $\mathrm{O}$ atom and the two end atoms and $\alpha$ is the bond angle at the center atom. Although the PESs are symmetric in
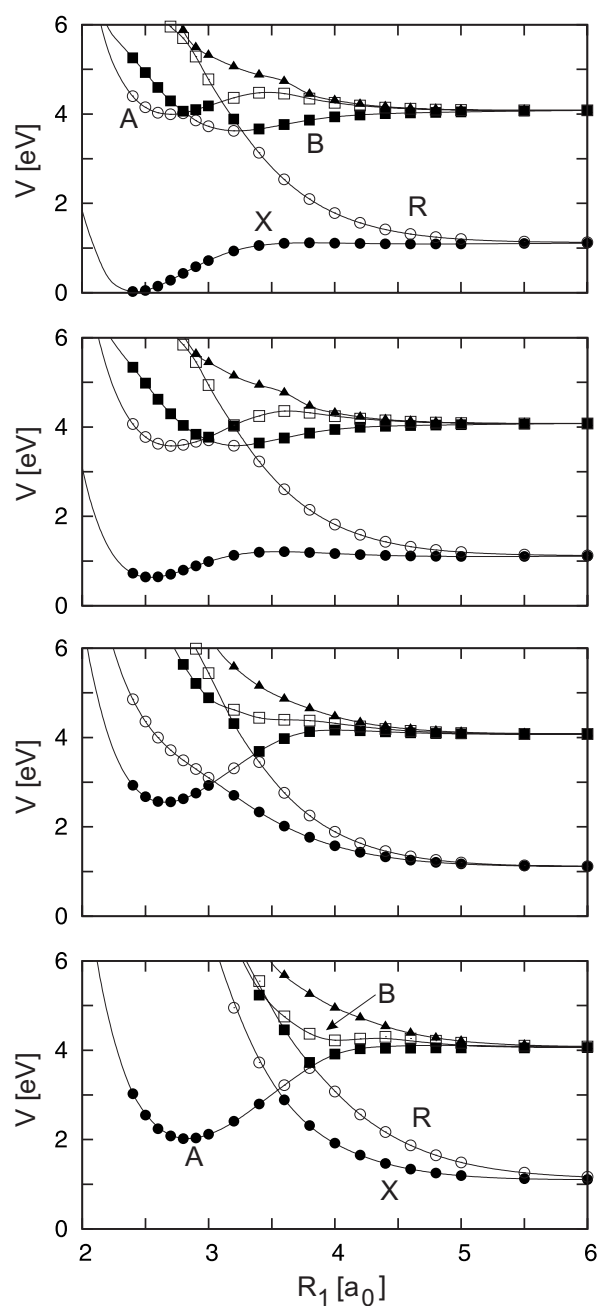

FIG. 1. Potential energies of the lowest five ${ }^{1} A^{\prime}$ states of ozone as functions of the dissociation bond length $R_{1}$ for several bond angles $\alpha\left(120^{\circ}, 100^{\circ}\right.$, $80^{\circ}$, and $60^{\circ}$ from top to bottom) and $\mathrm{O}_{2}$ bond length $R_{2}=2.40 a_{0}$. Energy normalization is such that $E=0$ corresponds to the minimum of the ground state. The symbols indicate adiabatic energies (shown only for $R_{1} \geq R_{2}$ ) and the lines represent diabatic potential curves labeled $\mathrm{X}, \mathrm{A}, \mathrm{B}$, and $\mathrm{R}$.

$R_{1}$ and $R_{2}$, we will term $R_{1}$ the dissociation coordinate and $R_{2}$ the $\mathrm{O}_{2}$ bond coordinate. Potential energies are calculated on a large coordinate grid. $R_{1}$ (in $a_{0}$ ) is varied in the range 1.8-3.0 with $\Delta R_{1}=0.1$, in the range 3.2-5.0 with $\Delta R_{1}=0.2$, and 5.5-8.0 with $\Delta R_{1}=0.5$. The grid in $R_{2}$ is $1.8-3.0$ with $\Delta R_{2}=0.1$ and 3.2-4.0 with $\Delta R_{2}=0.2$. The angle is varied between $60^{\circ}$ and $160^{\circ}$ with $\Delta \alpha=10^{\circ}$ and with $\alpha=85^{\circ}$ instead of $90^{\circ}$. Energies for angles below $60^{\circ}$ can be determined from $\alpha>60^{\circ}$. Electronic structure calculations are performed for $R_{2} \leq R_{1}$ only and energies for $R_{2}>R_{1}$ are obtained by symmetry. The grid in the present calculations extends considerably farther toward the three-body breakup than in the previous calculations. ${ }^{12}$ This is necessary for unraveling the dissociation mechanism yielding $\mathrm{O}_{2}$ products with very high internal energy. ${ }^{14}$ In order to highlight special features of the interaction potentials, some additional calculations are performed on finer coordinate grids. Throughout this publication the normalization of energy is such that $E=0$ corresponds to the equilibrium geometry in the ground electronic state of ozone.

In Figs. 1-3 we show one-dimensional cuts along the 

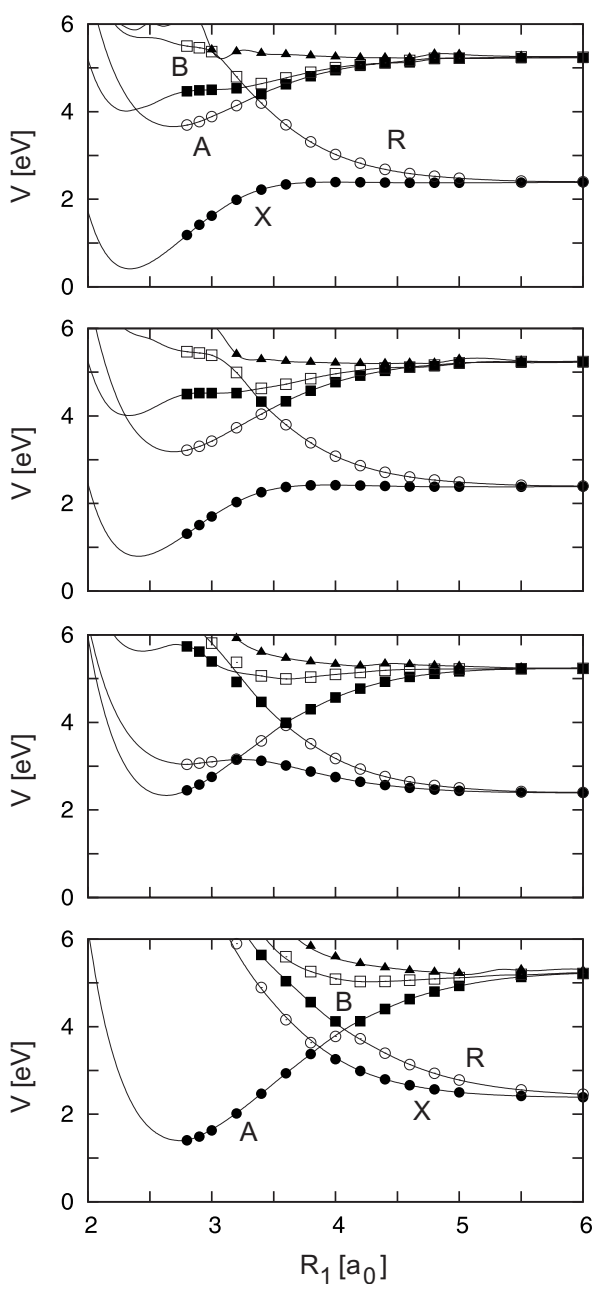

FIG. 2. The same as in Fig. 1 for $R_{2}=2.80 a_{0}$.

dissociation bond $R_{1}$ for selected $\alpha$ and $R_{2}$. The different symbols represent the adiabatic potential energies. These 12 cuts are chosen in order to illustrate the complexity of the potential energies and how the appearances of the various avoided crossings change with $R_{2}$ and $\alpha$. Cuts along the bond angle $\alpha$ were given in Ref. 27, for example. The density of electronic states (of the same symmetry) drastically increases when all three bond distances become large, i.e., when ozone dissociates into three $\mathrm{O}\left({ }^{3} P\right)$ atoms. ${ }^{14,27}$ The potentials shown in Fig. 3 for $R_{2}=3.2 a_{0}$ provide just an impression of this trend; with increasing $R_{2}$ all potentials approach the same dissociation limit. In the present work we calculated, because of limited computational power, only five ${ }^{1} A^{\prime}$ states, even though the next higher ones are energetically close for intermediate and large $R_{1}{ }^{14}$ The lowest five are sufficient for describing the main dissociation dynamics and the main part of experimental observations in the Huggins/Hartley band system.

In most of the cuts depicted in Figs. 1-3 two states are involved in an avoided crossing and the coupling between them can be described in a comparatively simple way (see below). However, the crossings move with $R_{2}$ and $\alpha$ and therefore there are regions where three states are almost degenerate. An example is highlighted in Fig. 4 for $\alpha=110^{\circ}$ and $R_{2}=2.60 a_{0}$. In the vicinity of this triple crossing the
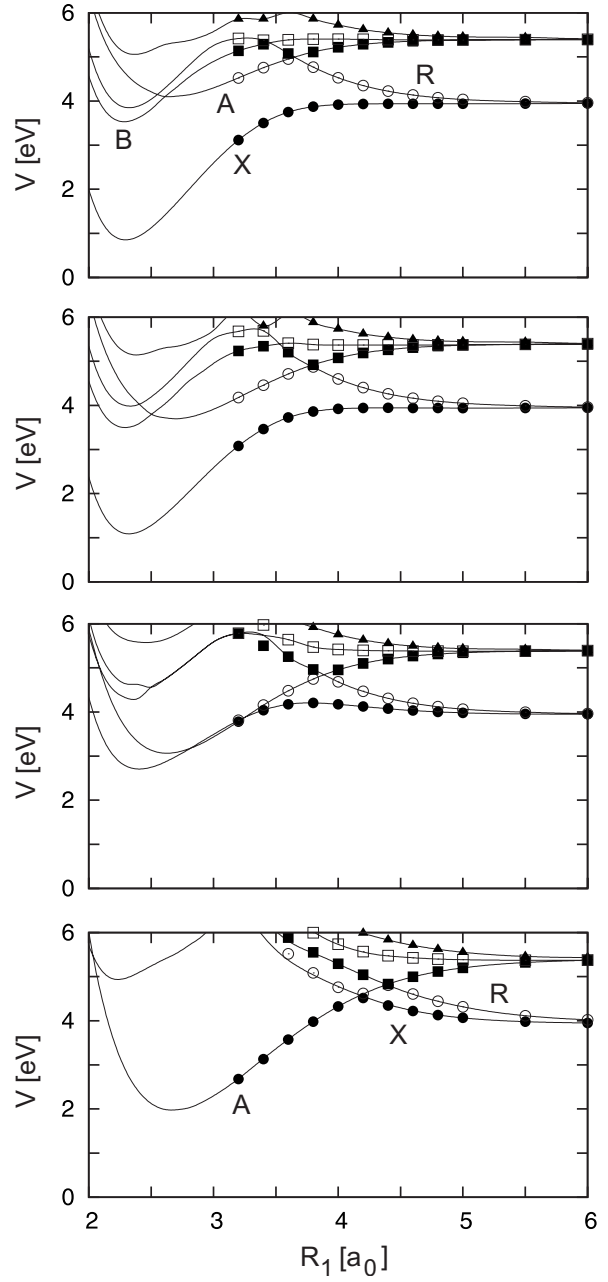

FIG. 3. The same as in Fig. 1 for $R_{2}=3.20 a_{0}$.

adiabatic energies show a complicated behavior, which can be resolved only with calculations on a fine grid. Avoided crossings between three states-the first, second, and third ${ }^{1} A^{\prime}$ states-also occur at small angles near $\alpha \approx 70^{\circ}$ (see below).

The energy separation between the two dissociation channels (1) and (2) is more or less equal for $R_{2}=2.4 a_{0}$ and $2.8 a_{0}$ (Figs. 1 and 2), but is significantly smaller for $R_{2}$

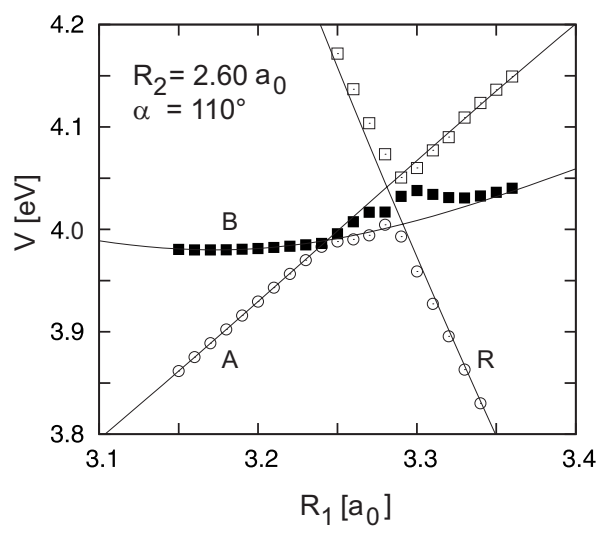

FIG. 4. Potential energies in the vicinity of the three-state crossing between states A, B, and R. $\alpha=110^{\circ}$ and $R_{2}=2.6 a_{0}$. The symbols indicate the adiabatic energies and the lines represent the diabatic potentials. 

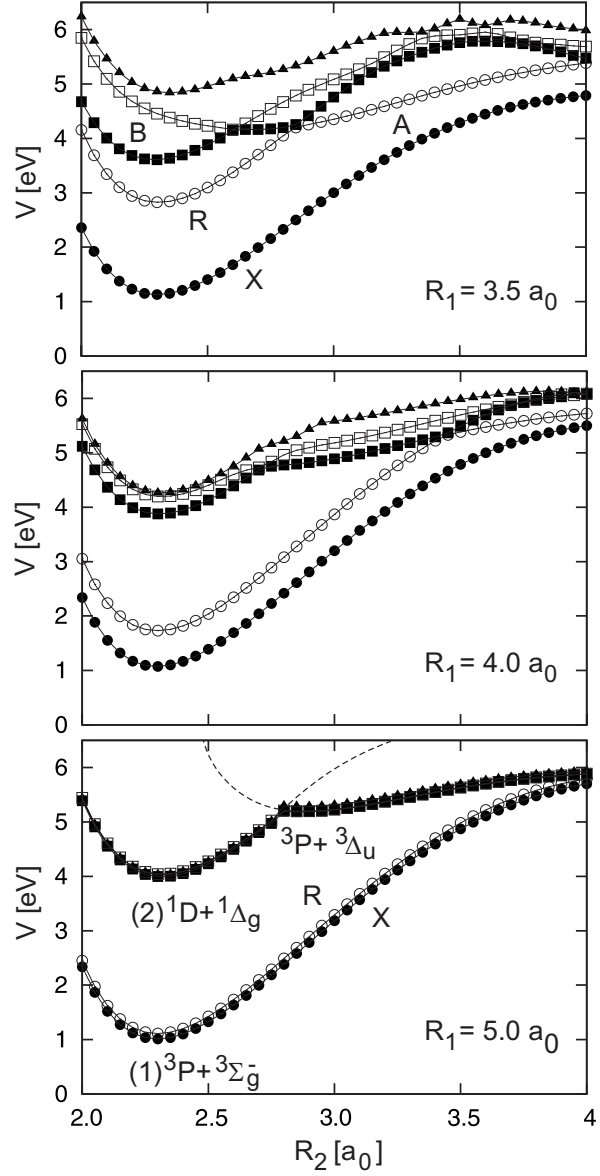

FIG. 5. Potential energies of the lowest five ${ }^{1} A^{\prime}$ states vs $\mathrm{O}_{2}$ bond length $R_{2}$ for three values of the dissociation bond length $R_{1}$ and $\alpha=100^{\circ}$. The symbols represent the adiabatic energies and $\mathrm{X}, \mathrm{A}, \mathrm{B}$, and $\mathrm{R}$ indicate the diabatic potentials. The two dashed lines in the lower panel schematically indicate the continuations of the potentials belonging to the singlet channel (2) and the excited triplet (Herzberg) channel, respectively. Several additional states are required to obtain a more complete picture (Ref. 14).

$=3.2 a_{0}$ (Fig. 3 ) and becomes gradually smaller with increasing $R_{2}$ (not shown here). This shift hints at a qualitative change of the PESs for large $\mathrm{O}_{2}$ bond lengths. In order to clarify the behavior at large $R_{2}$ we performed additional electronic structure calculations on a narrow grid of $R_{2}$ for several values of $R_{1}$ and $\alpha=100^{\circ}$ (Fig. 5). With increasing $R_{1}$ the potential cuts shown in Fig. 5 gradually turn into the $\mathrm{O}_{2}$ stretching potentials in the presence of $\mathrm{O}\left({ }^{3} P\right.$ ) (lower two curves) or $\mathrm{O}\left({ }^{1} D\right)$ (upper three curves), respectively. The potential cuts for $R_{1}=3.5 a_{0}$ show no peculiar behavior, except for several avoided crossings. For $R_{1}=4 a_{0}$ it is already apparent that the two lowest curves approach the triplet channel (1) while the three higher potential curves represent-for small bond distances $R_{2}$-the singlet channel (2). $\mathrm{O}_{2}\left(a^{1} \Delta_{g}\right)$ dissociates, like $\mathrm{O}_{2}\left(X^{3} \Sigma_{g}^{-}\right)$, into two $\mathrm{O}\left({ }^{3} P\right)$ atoms ${ }^{28}$ and therefore one expects the potential curves corresponding to channel (2) to approach a value which in the limit $R_{2} \rightarrow \infty$ is roughly $2 \mathrm{eV}$ higher than for channel (1). However, the $\mathrm{O}_{2}$ potential cuts in Fig. 5 for $R_{1} \geq 4 a_{0}$ approach a common limit at about $6 \mathrm{eV}$. The common asymptotic limit appears because at large $R_{2}$ the upper "diabatic" states acquire the electronic character of a different state of $\mathrm{O}_{2}$ correlating with $\mathrm{O}\left({ }^{3} \mathrm{P}\right)$.

As the $\mathrm{O}$ atom is gradually more detached from $\mathrm{O}_{2}$
$\left(R_{1}=4.0 a_{0}\right.$ and $5.0 a_{0}$ in Fig. 5) the energy splittings between the potential curves shrink and at the same time the "kink" at $R_{2} \approx 2.8 a_{0}$ becomes more distinct. This kink is caused by the intersection of the potential curve(s) representing channel (2), $\mathrm{O}\left({ }^{1} D\right)+\mathrm{O}_{2}\left({ }^{1} \Delta_{g}\right)$, and the lowest potential curve(s) for the excited triplet channel, $\mathrm{O}\left({ }^{3} P\right)+\mathrm{O}_{2}\left({ }^{3} \Delta_{u}\right)$, mentioned in the Introduction. State $A^{\prime}{ }^{3} \Delta_{u}$ is one of the Herzberg states of oxygen. ${ }^{29}$ Another one, $A^{3} \Sigma_{u}^{+}$, which is higher by only $630 \mathrm{~cm}^{-1}$, does not appear in Fig. 5. Many more "supermolecular" states of $\mathrm{O}-\mathrm{O}_{2}$ will have to be calculated before a complete picture emerges, including the repulsive branch of the Herzberg channel and the extension of channel (2) to large $R_{2} .{ }^{14}$ The crossing of the potentials for the singlet and the excited triplet channels for large $R_{1}$ is simply the consequence of the ${ }^{3} P-{ }^{1} D$ excitation of the sibling $\mathrm{O}$ atom; transitions between the two channels are not associated with this crossing. However, for finite $R_{1}$ all states have the same symmetry ${ }^{1} A^{\prime}$, the degeneracies of the potentials are lifted, the real crossings turn into avoided crossings, and nonadiabatic transitions do become possible. The implications for the UV photodissociation have been discussed elsewhere. ${ }^{14}$ Here, we consider the large- $R_{2}$ behavior of the potentials only to rationalize the shape of the B-state diabatic PES discussed below. The accessibility of the Herzberg channel for $\lambda<234 \mathrm{~nm}$ is an intriguing aspect of the UV excitation of ozone; however, it does not significantly affect the main part of the dissociation in the Hartley band.

\section{DIABATIC POTENTIAL ENERGY SURFACES}

Because of the several avoided crossings, smooth diabatic PESs are more useful for dynamics calculations than the adiabatic PESs; the latter would require narrow grids for accurate representations of the avoided crossings, not to mention the calculation of many nonadiabatic coupling matrix elements (NACMEs).$^{30}$ Diabatic PESs have been determined straightforwardly for the two lowest ${ }^{1} A^{\prime \prime}$ and the two lowest ${ }^{3} A^{\prime \prime}$ states of ozone with conical intersections in $C_{2 v}$ (Chappuis band and Wulf band, respectively). ${ }^{10,31}$ The construction of diabatic PESs relevant for the UV photodissociation is much more demanding for several reasons. (1) The avoided crossings occur far away from the $C_{2 v}$ symmetry plane. (2) There are regions of coordinate space where more than two states interact; in particular, many states have to be taken into account when all three $\mathrm{O}-\mathrm{O}$ bond distances are stretched as discussed at the end of Sec. II. (3) The pattern of avoided crossings changes noticeably with both $R_{2}$ and $\alpha$; for example, it is different for angles around $120^{\circ}$ ("open ozone") and $60^{\circ}$ ("cyclic ozone"). A rigorous diabatization of all relevant states, covering the entire coordinate space, is exceedingly complicated.

Here, we construct diabatic PESs for states X, B, A, and $\mathrm{R}$ as in the previous work of $\mathrm{Qu}$ et al. ${ }^{9}$ by simply collecting energies which apparently belong to the same diabatic state. The diabatization by eye is unambiguous and straightforward when the splitting of the adiabatic energies is relatively small at the avoided crossing and rapidly grows with increasing distance from the crossing; then it is obvious how to assign the calculated adiabatic energies to obtain smooth diabatic 
potentials. This is the case for the biggest part of the threedimensional coordinate space, especially along the main fragmentation route. The diabatization by eye gradually becomes more ambiguous, however, towards the three-body breakup when all three $\mathrm{O}-\mathrm{O}$ bond lengths are large. The potential cuts for the upper states in Fig. 3, for example, illustrate the increasing difficulty in assigning diabatic states. The problems become more severe when the $\mathrm{O}_{2}$ bond is even further stretched.

The selected diabatic potential energies are interpolated by three-dimensional cubic splines. The interpolated diabatic potential curves are also shown in Figs. 1-3. In most cases they smoothly connect the original potential energies. The uppermost state is not included in the diabatization procedure and this is the reason for the avoided crossing between the R-state PES and the highest one observed in some of the panels. This avoided crossing occurs predominantly near $C_{2 v}$ geometries. It is not relevant for the overall dissociation dynamics since the trajectories accessing the R-state PES quickly dissociate; the B- and R-state potentials cross far away from $C_{2 v}$. An ambiguity in the definition of the A- and B-state diabatic potentials is apparent in Fig. 1 for $\alpha=60^{\circ}$ and $4 a_{0}<R_{1}<5 a_{0}$. This angular region of the coordinate space is also not important for the dissociation dynamics; moreover, both states connect with the same product channel (2) and thus any transitions between states A and B would not affect the quantum yields. Figure 4 illustrates how the diabatic potentials approximate the adiabatic energies in the vicinity of the three-state crossing between states B, A, and $\mathrm{R}$. The grid from which the global diabatic potentials were constructed includes merely the points $R_{1}=3.2 a_{0}$ and $3.4 a_{0}$, i.e., the complicated region is excluded from the original grid.

In Fig. 6 we show two-dimensional representations of the diabatic PESs for states B, A, X, and R for fixed bond angle $\alpha=110^{\circ}$. The B-state PES has two identical nonsymmetric potential wells separated by a barrier in the $C_{2 v}$ plane. Excitation of the vibrational states supported by the potential wells gives rise to the weak Huggins band superimposed on the red branch of the Hartley band. ${ }^{12}$ The PES of the A-state has a symmetric potential well and, for this angle, a narrow transition state barrier for dissociation. At intermediate and large distances, $R_{1} \geq 4 a_{0}$, both the B- and the A-state PESs show a double-well potential behavior along the $\mathrm{O}_{2}$ bond distance $R_{2}$, which is due to the combination of the singlet channel and the excited triplet channel discussed above. Thus, for small $\mathrm{O}_{2}$ bond lengths $R_{2} \lesssim 2.8 a_{0}$ states $\mathrm{B}$ and A correlate with the singlet channel, while for large $R_{2}$ they correlate with the Herzberg states. ${ }^{32}$ The PES of the ground state $\mathrm{X}$ has a potential well of about one $\mathrm{eV}$ with a very small dissociation barrier that is hardly discernible in this representation. ${ }^{33}$ The R-state PES is strongly repulsive with a high rim along the symmetry line. Due to the interaction with higher states discussed above, its precise shape near the $C_{2 v}$ line is unclear. Both the X-and the R-state PESs correlate with the triplet channel (1). Figure 7 shows two-dimensional representations of the A, X, and R PESs for $\alpha=60^{\circ}$. The minimum of the A-state PES corresponds to cyclic ozone with $D_{3 h}$ symmetry. The other two potentials are strongly

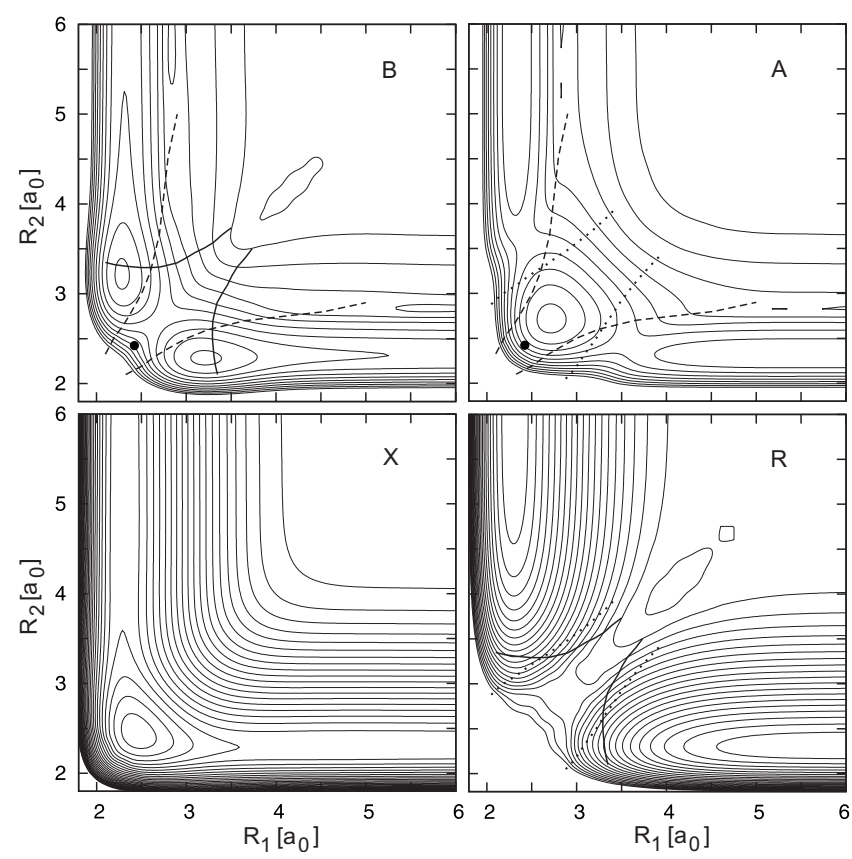

FIG. 6. Two-dimensional representations of the diabatic PESs for states B, $\mathrm{A}, \mathrm{X}$, and $\mathrm{R}$ for $\alpha=110^{\circ}$. The highest contour is $E=6 \mathrm{eV}$ and $\Delta E$ $=0.25 \mathrm{eV}$. The dots in the two upper panels mark the Franck-Condon point. The lines indicate the crossings of the diabatic potentials: (solid lines) $B / R$, (dashed lines) B/A, and (dotted lines) A/R.

repulsive for this bond angle (see also Figs. 1-3). The region of cyclic ozone is interesting for several reasons to be discussed below. With the diabatic PESs at hand it is easy to determine the crossings of the various diabatic states. Some of them are included in Figs. 6 and 7. Characteristic features of the states X, B, and A obtained from the interpolated PESs are given in Table I.

\section{DIABATIC COUPLING POTENTIALS}

In this section we describe the construction of the offdiagonal diabatic matrix elements $V_{k j}(k, j=\mathrm{B}, \mathrm{A}, \mathrm{R}$, or $\mathrm{X})$ that govern the coupling between the different diabatic states and therefore the singlet and triplet quantum yields. The largest effort is dedicated to the coupling between states B and $\mathrm{R}$, which is the most important one; the couplings between the other states are treated at a less detailed level.

\section{A. Coupling between states $B$ and $R$}

The construction of the coupling element $V_{\mathrm{BR}}$ follows several steps. In order to accurately determine the splitting of the adiabatic potentials, $V_{i}^{(a)}-V_{j}^{(a)}$, in the vicinity of the crossing seam, we first perform CASSCF and CI calculations on a dense grid along the dissociation bond $R_{1}$ for fixed $R_{2}$ and $\alpha$. The indices $i$ and $j$ are chosen so that the adiabatic potentials $V_{i}^{(a)}$ and $V_{j}^{(a)}$ correspond to the diabatic potentials $V_{\mathrm{B}}$ and $V_{\mathrm{R}}$, respectively; they vary with $R_{2}$ and $\alpha$. An example for $R_{2}=2.7 a_{0}$ and $\alpha=120^{\circ}$ is depicted in Fig. 8(a). The deviation of the lower curve from a linear behavior at $R_{1}$ $=3.32 a_{0}$ is the result of the crossing between states A and R, which is treated separately. One-dimensional interpolation of the adiabatic potential curves yields the crossing bond length $R_{1 x}$ (where $\left|V_{i}^{(a)}-V_{j}^{(a)}\right|$ has a minimum) and the energy sepa- 

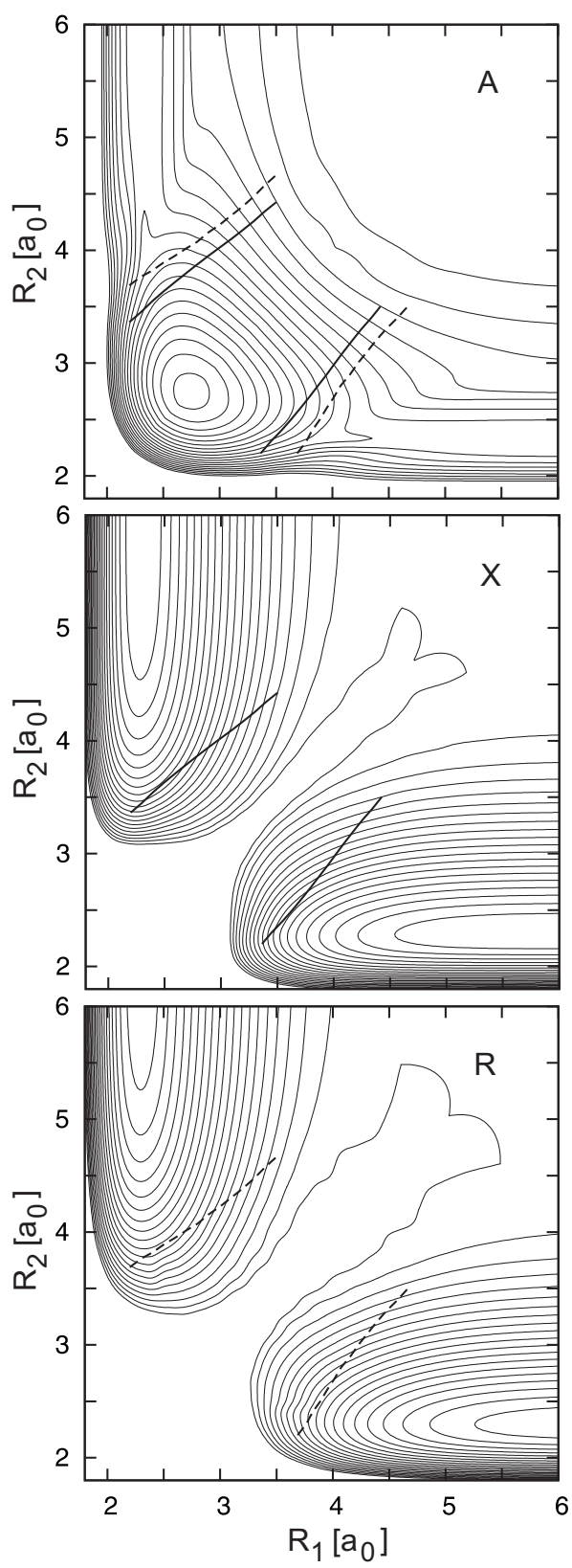

FIG. 7. Two-dimensional representations of the diabatic PESs of states A, $\mathrm{X}$, and $\mathrm{R}$ for $\alpha=60^{\circ}$. The highest contour is $E=6 \mathrm{eV}$ and $\Delta E=0.25 \mathrm{eV}$. The lines indicate the crossings of the diabatic potentials. Upper panel: $\mathrm{A} / \mathrm{X}$ (solid line) and A/R (dashed line). Middle panel: A/X. Lower panel: A/R.

ration at the crossing, $\Delta E_{x}$. Calculations of this kind are performed for $R_{2}=2.1,2.3,2.5,2.7,2.9,3.0$, and $3.2 a_{0}$ and $80^{\circ} \leq \alpha \leq 140^{\circ}$ with $\Delta \alpha=10^{\circ}$. Both $R_{1 x}$ and $\Delta E_{x}$ are interpolated by two-dimensional cubic splines.

In the second step we calculate- by numerical differentiation of the adiabatic electronic wave functions ${ }^{26}$ - the NACMEs in the vicinity of the crossing seam for fixed $R_{2}$ and $\alpha$, i.e.,

$$
D_{i j}^{R_{1}}\left(R_{1} \mid R_{2}, \alpha\right)=\left\langle\Xi_{i}^{(a)}\left|\frac{\partial}{\partial R_{1}}\right| \Xi_{j}^{(a)}\right\rangle .
$$

The $\Xi_{i}^{(a)}\left(R_{1}, R_{2}, \alpha\right)$ are the adiabatic electronic wave functions for states $i$ and $j$, which depend parametrically on the nuclear coordinates. Because these calculations are very time consuming at the CI level of theory, CASSCF wave functions are used. The NACME for $R_{2}=2.7 a_{0}$ and $\alpha=120^{\circ}$ is shown in Fig. 8(b). The width of $D_{i j}^{R_{1}}\left(R_{1} \mid R_{2}, \alpha\right)$ is unequivocally related to $\Delta E_{x}$. The smaller the adiabatic energy separation, the narrower the NACME and vice versa. Since $\Delta E_{x}$ can be very small, a very fine grid in $R_{1}$ is required. The NACMEs are mainly calculated for one angle, $\alpha=120^{\circ}$, and for all $R_{2}$ of the two-dimensional grid on which $\Delta E_{x}$ and $R_{1 x}$ are defined. Some of the NACMEs are also calculated by means of CI wave functions in order to test the applicability of CASSCF wave functions. The deviations are not significant. Since $R_{1 x}$ depends slightly on the level of theory, the NACMEs calculated with CASSCF and CI wave functions are somewhat shifted with respect to each other.

In the third step a coordinate dependent mixing angle $\varphi$ is determined by means of the NACMEs. The mixing angle is assumed to have the form ${ }^{31}$

$$
\varphi\left(R_{1}\right)=\frac{1}{2} \tan ^{-1}\left[a\left(R_{1 x}^{\prime}-R_{1}\right)\right]+\frac{\pi}{4},
$$

where the parameters $a\left(R_{2}, \alpha\right)$ and $R_{1 x}^{\prime}\left(R_{2}, \alpha\right)$ are determined by least squares fitting of $d \varphi / d R_{1}$ to the calculated NACME, that is,

$$
D^{R_{1}}\left(R_{1}\right)=-\frac{a}{2}\left\{1+\left[a\left(R_{1 x}^{\prime}-R_{1}\right)\right]^{2}\right\}^{-1} .
$$

The parameter $a$ is related to the width of the NACME. The full width at half maximum of $D^{R_{1}}$ is given by $\Delta R=2 / a$. The crossing radius $R_{1 x}^{\prime}$ can slightly differ from the $R_{1 x}$ obtained from the separation of the adiabatic potentials as described above. In Fig. 8(b) we compare the fitted NACME with the original one; the deviations are inconsequential. The mixing angle $\varphi$ is also shown; it asymptotically approaches $\pi / 2$ or 0 away from the crossing seam.

According to the general diabatization procedure ${ }^{30} V_{\mathrm{BR}}$ is defined by

$$
V_{\mathrm{BR}}\left(R_{1}, R_{2}, \alpha\right)=\cos \varphi \sin \varphi\left[V_{i}^{(a)}-V_{j}^{(a)}\right] .
$$

Figure 8(c) depicts $V_{\mathrm{BR}}\left(R_{1}\right)$ for $R_{2}=2.70 a_{0}$ and $\alpha=120^{\circ}$. It is nearly constant over the region where it is calculated. The

TABLE I. Characteristic features of the diabatic PESs for states X, B, and A.

\begin{tabular}{lccc}
\hline \hline & $\mathrm{X}$ & $\mathrm{B}$ & $\mathrm{A}$ \\
\hline$R_{1 \mathrm{e}}\left(a_{0}\right)$ & 2.422 & 3.210 & 2.740 \\
$R_{2 \mathrm{e}}\left(a_{0}\right)$ & 2.422 & 2.286 & 2.740 \\
$\alpha_{\mathrm{e}}(\mathrm{deg})$ & 116.6 & 106.9 & 60 \\
$V_{\mathrm{e}}(\mathrm{eV})$ & 0 & 3.440 & 1.372 \\
$V_{\text {vert. }}(\mathrm{eV})^{\mathrm{a}}$ & 0 & 5.040 & 4.272 \\
$V_{\text {diss. }}(\mathrm{eV})$ & 1.031 & $4.026^{\mathrm{b}}$ & $4.029^{\mathrm{b}}$ \\
$R_{\text {sad. }}\left(a_{0}\right)^{\mathrm{c}}$ & $\ldots$ & 2.69 & $\ldots$ \\
$\alpha_{\text {sad. }}(\mathrm{deg})^{\mathrm{c}}$ & $\ldots$ & 109.8 & $\ldots$ \\
$V_{\text {sad. }}(\mathrm{eV})^{\mathrm{c}}$ & $\ldots$ & 4.339 & $\ldots$ \\
\hline \hline
\end{tabular}

${ }^{\mathrm{a}}$ Vertical energies at the calculated X-state equilibrium.

${ }^{b}$ Differences due to inaccuracies of the electronic structure calculations, the diabatization, and the spline interpolation.

${ }^{\mathrm{c}}$ Saddle of the B-state PES. 


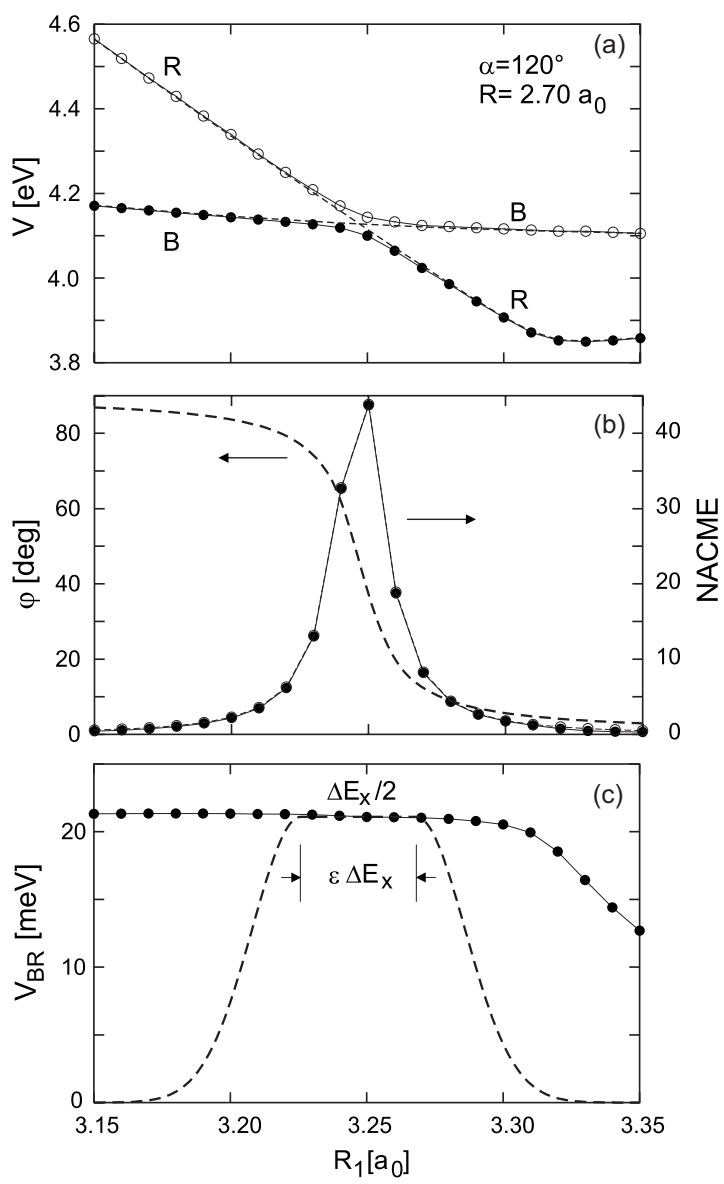

FIG. 8. (a) Adiabatic (solid lines and symbols) and diabatic (dashed lines) potentials near the $\mathrm{B} / \mathrm{R}$ crossing for $R_{2}=2.7 a_{0}$ and $\alpha=120^{\circ}$. (b) NACME (in $a_{0}^{-1}$, right-hand scale) and mixing angle $\varphi$ (left-hand scale, thick dashed line). Also shown is $d \varphi / d R_{1}$ (in rad. $a_{0}^{-1}$, open circles); it agrees almost exactly with the NACME. (c) Coupling matrix element $V_{\mathrm{BR}}$ obtained from Eq. (6) (filled circles) and from the model defined in Eq. (7) (dashed line). $\epsilon \Delta E_{x}$ is the width of the region over which $V_{\mathrm{BR}}$ is set to the constant $\Delta E_{x} / 2$. All data in this figure are obtained by CASSCF calculations.

trigonometric factor in Eq. (6) goes to zero on both sides of the crossing. On the other hand, $\left|V_{i}^{(a)}-V_{j}^{(a)}\right|$ increases with $\left|R_{1}-R_{1 x}\right|$ and thus compensates the decaying $\cos \varphi \sin \varphi$ term. The sharp drop beyond $3.3 a_{0}$ again reflects the avoided crossing between states A and R [see Fig. 8(a)]. From $\varphi\left(R_{1 x}\right)=\pi / 4$ it follows that $V_{\mathrm{BR}}\left(R_{1 x}\right)=\Delta E_{x} / 2$ at the crossing. Similar analyses are performed for other combinations of $R_{2}$ and $\alpha$ and qualitatively very similar results are obtained.

In the local diabatization scheme used here, the diabatic potentials must match the adiabatic ones outside the crossing zone so the coupling potential needs to vanish far from the crossing. ${ }^{34}$ Since the coupling potentials constructed from the NACMEs are nearly constant at $\Delta E_{x} / 2$ over a wide region around the crossing, in a fourth step we represent $V_{\mathrm{BR}}$ with the model

$$
\begin{aligned}
& V_{\mathrm{BR}}\left(R_{1}\right) \\
& \quad=\frac{\Delta E_{x}}{2} \begin{cases}\mathrm{e}^{-\delta\left[R_{1}-\left(R_{1 x}-b / 2\right)\right]^{2}} & R_{1}<R_{1 x}-b / 2 \\
1 & R_{1 x}-b / 2 \leq R_{1} \leq R_{1 x}+b / 2 \\
\mathrm{e}^{-\delta\left[R_{1}-\left(R_{1 x}+b / 2\right)\right]^{2}} & R_{1}>R_{1 x}+b / 2,\end{cases}
\end{aligned}
$$

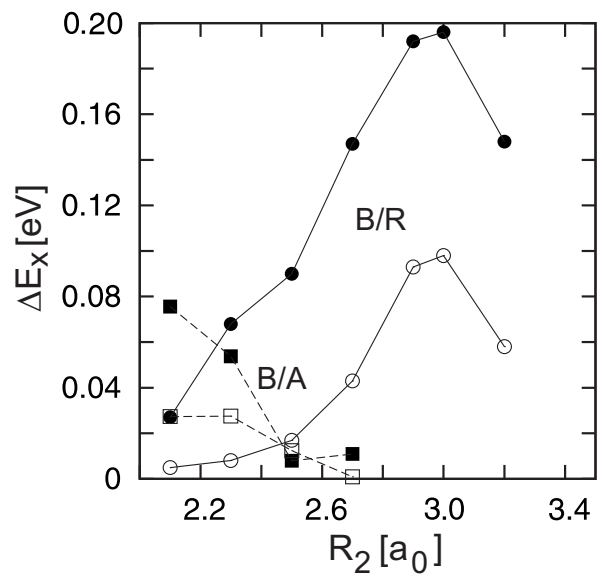

FIG. 9. Adiabatic energy separation $\Delta E_{x}$ as function of $R_{2}$ for the $\mathrm{B} / \mathrm{R}$ crossing (circles) and the $\mathrm{B} / \mathrm{A}$ crossing (squares); $\alpha=100^{\circ}$ (filled symbols) and $\alpha=120^{\circ}$ (open symbols).

where the range parameter $b$ is assumed to be proportional to $\Delta E_{x}$, i.e., $b=\epsilon \Delta E_{x}$. The proportionality parameter $\epsilon$ has units $a_{0} / \mathrm{eV}$. The other parameter, $\delta$, determines the width of the cutoff functions on both sides of the constant region. It is assumed to be related to $\Delta E_{x}$ through

$$
\delta=\frac{-4 \ln \frac{1}{2}}{\left(\Delta E_{x}\right)^{2}}\left(\frac{\mathrm{eV}}{a_{0}}\right)^{2}
$$

The half width at half maximum of the cutoff functions is proportional to $\Delta E_{x} / 2$. The smaller $\Delta E_{x}$ the faster the decay and vice versa. The expression for $V_{\mathrm{BR}}$ is symmetrized with respect to the interchange of $R_{1}$ and $R_{2}$ by taking the larger bond distance as $R_{1}$ and the smaller one as $R_{2}$. An example of the final $V_{\mathrm{BR}}$ used in the dynamics calculations is shown in Fig. 8(c).

The two main parameters of this model are $\Delta E_{x}\left(R_{2}, \alpha\right)$ and $R_{1 x}\left(R_{2}, \alpha\right)$. They are determined by the additional CI calculations on the narrow grid in $R_{1}$ described above. The $R_{1 x}$ obtained from the CI calculations and from the analytical PESs are almost identical. The crossing seam cuts right through the well of the B-state PES and runs more or less perpendicular to the dissociation path (Fig. 6). The energy separation $\Delta E_{x}$ varies strongly with $R_{2}$ as illustrated in Fig. 9 for two bond angles. It is small for small $\mathrm{O}_{2}$ bond lengths, i.e., around the equilibrium, and then rapidly increases to a maximum around $R_{2}=2.9 a_{0}$. Beyond the maximum $\Delta E_{x}\left(R_{2}\right)$ decreases again. A very small separation between the adiabatic potentials indicates a more diabatic dynamics while a large $\Delta E_{x}$ points to an adiabatic dynamical behavior. The strong dependence of $\Delta E_{x}\left(R_{2}\right)$ on $R_{2}$ is important for the dissociation dynamics, especially the distribution of the available energy in the triplet channel. ${ }^{20}$ The only free parameter is $\epsilon$ in the definition of the range parameter $b$; it will be adjusted by comparing the results of dynamical calculations with experimental data, primarily the quantum yield for the triplet channel. The parameter $\delta$ is fixed by Eq. (8) throughout the calculations. 

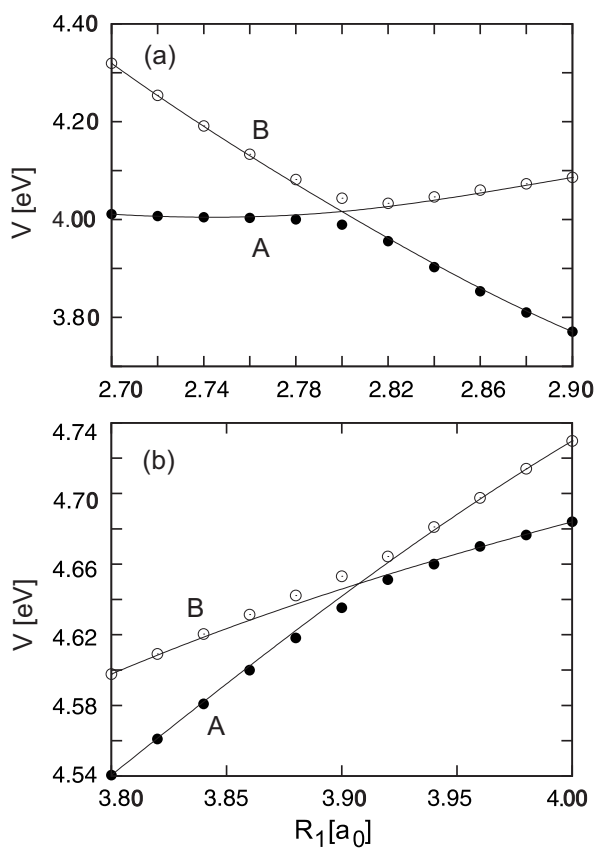

FIG. 10. Adiabatic (symbols) and diabatic (lines) potential energies in the vicinity of the $\mathrm{B} / \mathrm{A}$ crossing for $\alpha=100^{\circ} ; R_{2}=2.3 a_{0}$ (a) and $R_{2}=2.7 a_{0}$ (b).

\section{B. Coupling between states B and A}

The direction of the $\mathrm{B} / \mathrm{A}$ crossing seam is quite different from that of the $\mathrm{B} / \mathrm{R}$ crossing. While $R_{1 x}$ depends only slightly on $R_{2}$ for $\mathrm{B} / \mathrm{R}$, it shows a very strong $R_{2}$ dependence for B/A (Fig. 6). The B and A PESs cross each other near the Franck-Condon (FC) region, but as $R_{2}$ increases the crossing shifts further into the exit channel. The analytical model developed for $V_{\mathrm{BR}}$ and defined in Eq. (7) is also used for $V_{\mathrm{BA}}$. The parameters $R_{1 x}$ and $\Delta E_{x}$ are determined from additional CI calculations on a fine grid in $R_{1}\left(\Delta R_{1}=0.01 a_{0}-0.02 a_{0}\right)$ for $R_{2}=2.1-2.9 a_{0} \quad\left(\Delta R_{2}=0.2 a_{0}\right)$ and $\alpha=90^{\circ}-140^{\circ} \quad(\Delta \alpha$ $=10^{\circ}$ ) and interpolated by cubic splines. NACMEs are not explicitly calculated. The parameter $\delta$ has the same relationship to $\Delta E_{x}$ as for the $\mathrm{B} / \mathrm{R}$ crossing, Eq. (8), and $\epsilon$ $=1 a_{0} / \mathrm{eV}$, if not stated otherwise.

Figure 10 depicts the $\mathrm{B} / \mathrm{A}$ crossing behavior for $\alpha$ $=100^{\circ}$ and two values of $R_{2}$. At the smaller $\mathrm{O}_{2}$ bond length the two potentials intersect at a relatively large angle $\left(\Delta E / \Delta R_{1} \approx 3 \mathrm{eV} / a_{0}\right)$, while for $R_{2}=2.7 a_{0}$ the angle is considerably smaller $\left(\Delta E / \Delta R_{1} \approx 0.5 \mathrm{eV} / a_{0}\right)$. When $R_{2}$ increases the crossing point is further shifted to larger $R_{1}$ and eventually the two potentials do not cross at all. For $R_{2}$ $=2.8 a_{0}$ and $\alpha=100^{\circ}$, for example, a real crossing cannot be identified in Fig. 2. The separation of the adiabatic potentials at the crossing, $\Delta E_{x}$, is depicted in Fig. 9 as a function of $R_{2}$ for $\alpha=100^{\circ}$ and $120^{\circ}$. For both angles it has a maximum at small $R_{2}$ and then decreases to very small values between $2.5 a_{0}$ and $2.7 a_{0} . \Delta E_{x}$ for the $\mathrm{B} / \mathrm{A}$ crossing thus has a quite different behavior than $\Delta E_{x}$ for the $\mathrm{B} / \mathrm{R}$ crossing. The $\mathrm{B} / \mathrm{A}$ coupling is smallest for angles $100^{\circ}<\alpha<120^{\circ}$, which is the range sampled predominantly in the first moments of the dissociation. It increases, however, both at smaller and at larger bond angles. Since the vast majority of trajectories starting on the B-state PES near the FC point will cross the
B/A intersection with $R_{2}>2.4 a_{0}$, the probability for transitions to the A-state is expected to be very small and the TSH calculations confirm this expectation.

\section{Coupling between states $A$ and $X$}

The two lowest ${ }^{1} A^{\prime}$ states, which in the $C_{2 v}$ point group both have ${ }^{1} A_{1}$ symmetry, have an avoided crossing near $\alpha$ $=85^{\circ}{ }^{35-43}$ This crossing can be imagined in Figs. $1-3$ by comparison of the potential cuts for $\alpha=100^{\circ}$ and $80^{\circ}$. The ground state $\mathrm{X}$ becomes more repulsive while the A-state PES becomes more attractive as $\alpha$ is lowered. The $\mathrm{X}$ state correlates with open ozone and dissociation channel (1) while A correlates with the cyclic form of ozone at $\alpha=60^{\circ}$ and the excited channel (2). The crossing near $85^{\circ}$ leads to a considerable complexity of the PESs when the angle changes from about $110^{\circ}$ to $60^{\circ}$.

Xantheas et al $^{44}$ identified an "accidental conical intersection" in $C_{2 v}$ which was subsequently analyzed in great detail by Ruedenberg and co-workers. ${ }^{37,39,45,46}$ The term "accidental" was used because two states with like symmetry usually are not degenerate. The nonadiabatic coupling in the vicinity of this conical intersection (CI) was determined by Atchity and Ruedenberg ${ }^{47}$ and Sukumar and Peyerimhoff. ${ }^{48}$ At the level of the electronic structure calculations applied in the present study, the accidental CI occurs at $R_{1}=R_{2}$ $=2.95 a_{0}$ and $\alpha=82.0^{\circ}$. The energy separation between the two states at this geometry is $0.001 \mathrm{eV}$; a more refined search would further diminish this splitting.

The structure of the potential energies at small angles is even further complicated by the repulsive R-state, which for angles near $70^{\circ}$ has an avoided crossing with the X-state. Actually, there is another point in the coordinate space where three states-X, A, and R-cross: $\alpha \approx 70^{\circ}, R_{1} \approx 3.45 a_{0}$, and $R_{2} \approx 2.4 a_{0}$. For $\alpha=60^{\circ}$ and $80^{\circ}$ the X-and R-state potentials are well separated (Figs. 1-3). This avoided crossing between $\mathrm{X}$ and $\mathrm{R}$ is not considered in the diabatization procedure.

The coupling between states $\mathrm{A}$ and $\mathrm{X}$ is relevant for the dissociation path $\mathrm{B} \rightarrow \mathrm{A} \rightarrow \mathrm{X}$ suggested by Brouard et al. ${ }^{49}$ for explaining the very slow $\mathrm{O}\left({ }^{3} P\right)$ products in the triplet channel. The $\mathrm{A} / \mathrm{X}$ coupling is also important for the creation of cyclic ozone, for example, by isomerization from the $C_{2 v}$ to the $D_{3 h}$ well employing tailored laser pulses as put forward by Artamonov et al. ${ }^{50}$ and Kurosaki et al. ${ }^{51}$ Calculations using only the lowest adiabatic PES are unrealistic.

For $\alpha=60^{\circ}$ the crossings of diabatic states $\mathrm{X}$ and A occur at geometries well separated from the $C_{2 v}$ symmetry line, near the entrance to the product channels (Fig. 7). With increasing angle the crossing seam gradually shifts toward $C_{2 v}$ geometries and becomes more confined to small $\mathrm{O}-\mathrm{O}$ bond lengths. The crossings vanish for $\alpha \geq 87^{\circ}$, i.e., transitions between $\mathrm{X}$ and $\mathrm{A}$ are restricted mainly to the region of cyclic ozone.

In order to characterize the coupling strength between states $\mathrm{A}$ and $\mathrm{X}$ we performed electronic structure calculations on a narrow grid of $R_{1}\left(\Delta R_{1}=0.02 a_{0}\right)$ for $\alpha=60^{\circ}, 70^{\circ}$, and $80^{\circ}$ and different $\mathrm{O}_{2}$ bond lengths $R_{2}$. From these calculations we extracted the separation $\Delta E_{x}$ at the avoided crossing 


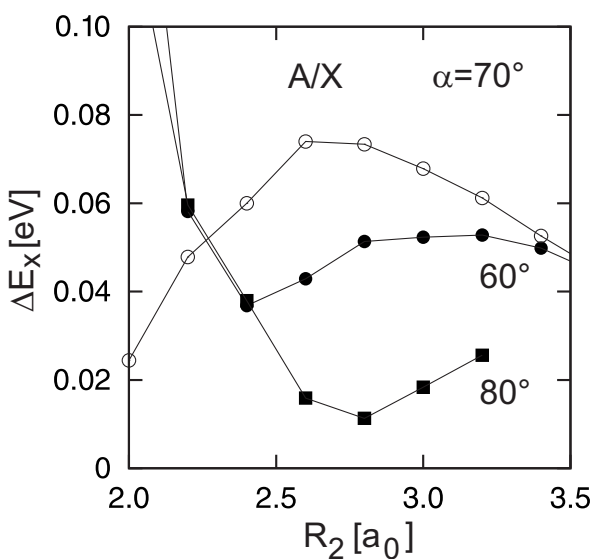

FIG. 11. Adiabatic energy separation $\Delta E_{x}$ for the $\mathrm{A} / \mathrm{X}$ crossing as function of $R_{2}$.

between $\mathrm{X}$ and $\mathrm{A}$, which serves as a measure of the coupling strength as already discussed for the $\mathrm{B} / \mathrm{R}$ and $\mathrm{B} / \mathrm{A}$ crossings. The adiabatic energy separation $\Delta E_{x}$, depicted in Fig. 11, shows a strong angle dependence. The minimum for $\alpha=80^{\circ}$ around $R_{2}=2.8 a_{0}$ reflects the tail of the $\mathrm{CI}$ at $82^{\circ}$ and $R_{1}=R_{2}=2.95 a_{0}$. The magnitude of $\Delta E_{x}$ is small for all three angles, indicating a predominantly diabatic behavior.

The population of state A via transitions from $\mathrm{B}$ is of the order of merely $1 \%$ (see below). Since the A/X coupling is also small, the net population transfer from $\mathrm{B}$ to $\mathrm{X}$ via $\mathrm{A}$ is expected to be negligible. For this reason we do not construct an off-diagonal element $V_{\mathrm{AX}}$ that reflects all details of the coordinate dependence of $\Delta E_{x}$ and $R_{1 x}$. We instead approximate $V_{\mathrm{AX}}$ by a Lorentzian function in the energy separation between $\mathrm{A}$ and $\mathrm{X}$,

$$
V_{\mathrm{AX}}\left(R_{1}, R_{2}, \alpha\right)=\frac{\overline{\Delta E}_{x}}{2} \frac{\kappa^{2}}{\left(V_{A}-V_{X}\right)^{2}+\kappa^{2}},
$$

where $\overline{\Delta E}_{x}$ is an average value, $0.05 \mathrm{eV}$ in the dynamics calculations. Transitions are confined to regions where the energy difference between $\mathrm{A}$ and $\mathrm{X}$ is small.

\section{Coupling between states $A$ and $R$}

The repulsive R-state potential, which leads to ground state products, crosses the A-state potential for the entire ranges of $R_{2}$ and $\alpha$ relevant for the dissociation process. In this sense it is qualitatively similar to the $\mathrm{B} / \mathrm{R}$ crossing. The crossing occurs predominantly at large $R_{1}$ bond lengths for both open and cyclic ozone (Figs. 6 and 7). In order to determine the splitting $\Delta E_{x}$ of the adiabatic potentials we performed additional electronic structure calculations on a narrow grid of $R_{1}\left(\Delta R_{1}=0.02 a_{0}\right)$ for five angles $\left(\alpha=60^{\circ}, 80^{\circ}\right.$, $100^{\circ}, 120^{\circ}$, and $140^{\circ}$ ) and different $\mathrm{O}_{2}$ bond lengths $R_{2}$ ranging from $2.0 a_{0}$ to $3.8 a_{0}$. Compared to the $\mathrm{B} / \mathrm{R}$ crossing, $\Delta E_{x}$ is small (Fig. 12); the maximum is about $0.05 \mathrm{eV}$ in the region of open ozone and $0.07 \mathrm{eV}$ around cyclic ozone. The population transfer from $\mathrm{B}$ to $\mathrm{R}$ via state $\mathrm{A}$ is expected to be small and therefore we again abstain from a detailed description of the coordinate dependence of the off-diagonal $A / R$ diabatic potential and represent $V_{\mathrm{AR}}$ by a Lorentzian function similar to Eq. (9) with an average $\overline{\Delta E_{x}}=0.04 \mathrm{eV}$.

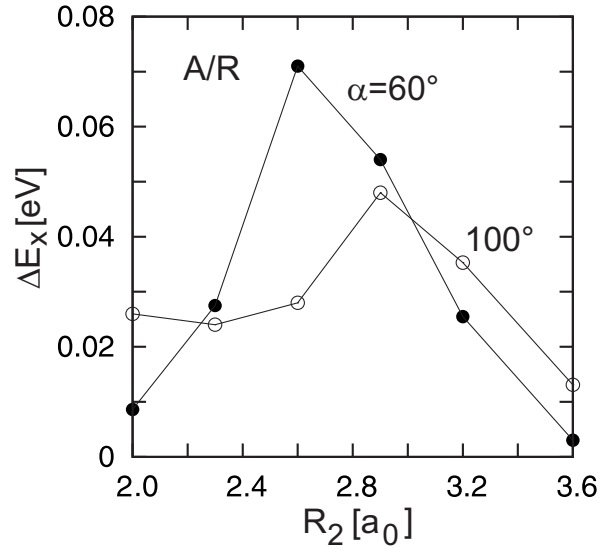

FIG. 12. Adiabatic energy separation $\Delta E_{x}$ for the $\mathrm{A} / \mathrm{R}$ crossing as function of $R_{2}$.

\section{TRAJECTORY SURFACE HOPPING CALCULATIONS}

The classical calculations (for total angular momentum $J=0)$ are performed by means of the symmetric Jacobi coordinates $R$ (distance from the central $\mathrm{O}$ atom to the center-ofmass of the two end atoms), $r$ (distance between the two end atoms), and $\gamma$ (angle between $\mathbf{R}$ and $\mathbf{r}$ ). Trajectories are started on the B-state PES with coordinates and momenta randomly selected in the six-dimensional phase space; only phase space points with the correct energy $( \pm 0.01 \mathrm{eV})$ are accepted. According to the classical theory of photodissociation ${ }^{30}$ each trajectory is weighted by the distribution function $\left|\Psi_{(000)}(R, r, \gamma)\right|^{2}\left|\Phi_{(000)}(R, r, \gamma)\right|^{2}$ multiplied by the square of the $\mathrm{X} \rightarrow \mathrm{B}$ transition dipole moment (TDM) function $\mu_{\mathrm{XB}} . \Psi_{(000)}$ is the wavefunction of the ground vibrational state in $\mathrm{X}$ and $\Phi_{(000)}$ is the corresponding momentum wavefunction. The latter is approximated by a threedimensional Gaussian centered at zero with widths derived from the widths of the coordinate wavefunction. ${ }^{30}$ The TDM is taken from the work of $\mathrm{Qu}$ et al. ${ }^{12}$

Transitions among the electronic states are modeled by the fewest switches surface hopping method of Tully, ${ }^{52}$ implemented in terms of electronic state amplitudes $a_{k}(t), k$ $=1-4$, rather than the electronic density matrix. The $a_{k}(t)$ are propagated in the interaction representation according to

$$
\frac{d a_{k}}{d t}=\frac{-i}{\hbar} \sum_{j \neq k} a_{j} \mathrm{e}^{i \phi_{k j} V_{k j}}
$$

where the $V_{k j}, k \neq j$, are the off-diagonal diabatic potential matrix elements $V_{\mathrm{BR}}, V_{\mathrm{BA}}$, etc. The nuclear potential energy along a trajectory is given by one of the diabatic PESs $V_{k}$, i.e., $V_{\mathrm{B}}, V_{\mathrm{R}}$, etc. The phases $\phi_{k j}$ are defined by

$$
\frac{d \phi_{k j}}{d t}=\frac{V_{k}-V_{j}}{\hbar} .
$$

The propagation begins on a single surface with $a_{k}=1$ for that surface (usually the B-state PES) and $a_{k}=0$ for all others. At the beginning of each trajectory all phases are initialized to zero. In Eq. (10) it is assumed that the "residual" kinetic energy couplings, the NACMEs defined in Eq. (3) for the diabatic states, are negligible. 
Along the trajectory the nuclei move according to Hamilton's equations on the current potential surface. The eight differential equations for the amplitudes, split into real and imaginary parts, and the six differential equations for the phases are combined with the six equations of motion for the three nuclear degrees of freedom to form a single system of up to 20 coupled equations. The nuclear and electronic sections are coupled because of the coordinate dependence of the potentials $V_{k}$ and the couplings $V_{k j}$.

After each numerical integration step $n$, the electronic population in the current state, $p_{n}=|a|^{2}$, is computed and if it is less than $p_{n-1}$, a transition to another state is possible. The probability for actually making a transition at this step $n$ is then $p_{\text {hop }}=\left(p_{n-1}-p_{n}\right) / p_{n-1}$. If a random number $\xi \in[0,1)$ is smaller than $p_{\text {hop }}$ then a hop should be attempted. The candidate destination states are those whose electronic populations increased during step $n$. For each of those states we compute the increase in population $\Delta p_{j}=\left|a_{j}^{(n)}\right|^{2}-\left|a_{j}^{(n-1)}\right|^{2}$. The fraction of $p_{\text {hop }}$ attributable to state $j$ is $f_{j}=\Delta p_{j} / \Sigma_{j} \Delta p_{j}$, where the sum runs over all the candidate states. Then, we consider each candidate state in turn. If $\xi \leq p_{\text {hop }} f_{j}$, we attempt to switch the current state to state $j$. If $\xi>p_{\text {hop }} f_{j}$ but $\xi$ $<p_{\text {hop }}\left(f_{j}+f_{k}\right)$, we attempt to switch to surface $k$, and so on.

At a hop, since the potential energies of the initial and final states are not usually equal, an adjustment must be made to the momenta to keep the total energy constant. We use a particularly simple procedure for that adjustment. On an "upward" hop we require that one-third of the necessary change in total kinetic energy be removed from each of the Jacobi momenta, and if any one of them is not large enough for that adjustment, the hop attempt is abandoned. Similarly, on "downward" hops the excess total kinetic energy is equally distributed among the three momenta. This procedure has the technical advantage that no spatial derivatives of the coupling potentials are required during the calculation. For some problems this equal-adjustment procedure might cause more frustrated hops than the usual prescription, ${ }^{52}$ and we discuss its influence in Sec. II. If the hop is successful, the candidate surface becomes the current one and the nuclei then move under its influence. The electronic part of the propagation continues as before; none of the electronic state amplitudes change at a hop.

The TSH calculations are fast, and comparison with accompanying wave packet calculations shows that they are trustworthy. Therefore they are well suited for investigating the influence of the parameters of the coupling potentials on, e.g., the quantum yields. In addition, they allow us to determine the regions of coordinate space where most transitions occur.

\section{RESULTS}

The coupling of state $\mathrm{B}$ to $\mathrm{R}$ is by far the most important one. We will, therefore, first present the results of two-state TSH calculations including only $\mathrm{B}$ and $\mathrm{R}$, before discussing four-state calculations. The results of the two-state calculations are obtained with typically 25000 trajectories, while 200000 trajectories are run in the four-state studies.

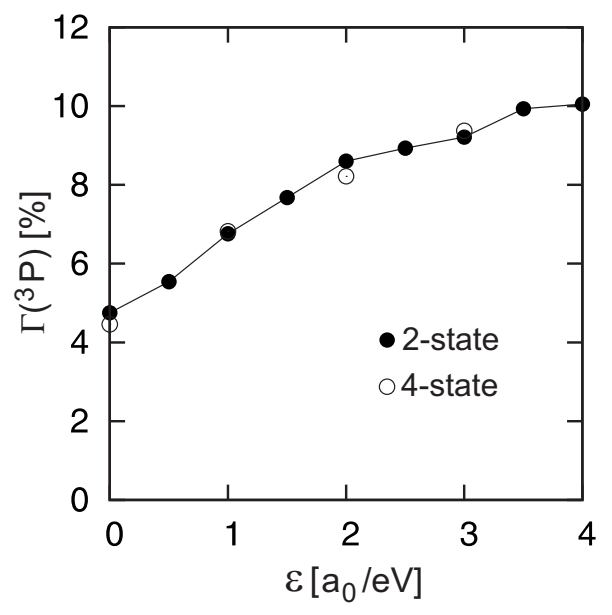

FIG. 13. Triplet channel yield $\Gamma\left({ }^{3} P\right)$ as function of the parameter $\epsilon$, used in Eq. (7) for $V_{\mathrm{BR}}$, for $257.1 \mathrm{~nm}$. Comparison of two-state $(\mathrm{B}, \mathrm{R})$ and four-state $(\mathrm{B}, \mathrm{R}, \mathrm{A}, \mathrm{X}) \mathrm{TSH}$ calculations.

\section{A. Two-state calculations: $B$ and $\mathbf{R}$ \\ 1. Sensitivity to coupling parameters}

The coupling element $V_{\mathrm{BR}}$ contains one free parameter, $\epsilon$, which determines the coordinate range over which the coupling is constant [Eq. (7) and Fig. 8]. For a fixed energy of $E=5 \mathrm{eV}$ corresponding to $\lambda=257.1 \mathrm{~nm}$ we determine the quantum yield $\Gamma\left({ }^{3} P\right)$ as function of $\epsilon$ (Fig. 13). It varies from about $5 \%$ at $\epsilon=0 a_{0} / \mathrm{eV}$ to $10 \%$ at $\epsilon=4 a_{0} / \mathrm{eV}$. Note that $\epsilon$ $=0$ does not mean that the coupling is zero; merely the constant range is shrunk to zero. The coupling element for $\epsilon$ $=0$ is a Gaussian function with a width proportional to $\Delta E_{x}$. As $\epsilon$ increases the gradient of $\Gamma\left({ }^{3} P\right)$ becomes gradually smaller, i.e., the quantum yield seems to saturate. Extending the range of $V_{\mathrm{BR}}$ in the direction of the dissociation coordinate $R_{1}$ is counterbalanced by the increase in the energy separation $\left|V_{\mathrm{B}}-V_{\mathrm{R}}\right|$ away from the crossing seam; when the energy mismatch between the states gets larger, transitions become more and more unlikely.

In order to check the sensitivity on the magnitude of $V_{\mathrm{BR}}$ we perform calculations for two values of $\epsilon$, in which $V_{\mathrm{BR}}$ is multiplied by a constant factor $\chi$ ranging from 0.1 to 2.0 (Fig. 14). $\Gamma\left({ }^{3} P\right)$ increases linearly for $\chi>0.5$ or so. For very

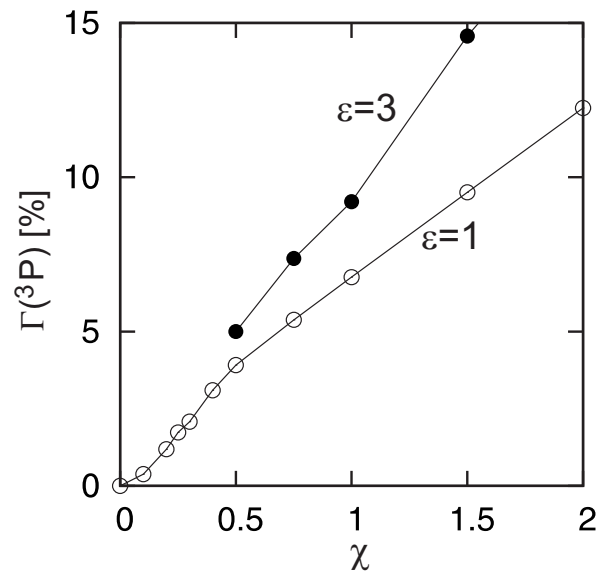

FIG. 14. Triplet channel yield $\Gamma\left({ }^{3} P\right)$ as function of an overall scaling factor $\chi$ for $257.1 \mathrm{~nm}$ and two values of $\epsilon$ (in $a_{0} / \mathrm{eV}$ ). 
small coupling potentials, $\chi \leqq 0.4$, however, the triplet quantum yield shows a more quadratic dependence as illustrated for $\epsilon=1.0$ in Fig. 14. A quadratic increase with the coupling strength is expected from perturbation theory.

The measured quantum yield for the singlet channel (2), $\Gamma\left({ }^{1} D\right)$, near $257 \mathrm{~nm}$ is of the order of $0.90-0.94 .{ }^{53}$ Considering $\Gamma\left({ }^{3} P\right)=1-\Gamma\left({ }^{1} D\right)$ gives a triplet yield of $0.06-0.10$, which is compatible with almost the entire variation of the calculated yield with $\epsilon$ in Fig. 13; with other words, $\epsilon$ is not a crucial parameter. In what follows we will use the value $\epsilon=1 a_{0} / \mathrm{eV}$. As we will discuss elsewhere, the shape of the kinetic energy distribution in the triplet channel is also affected by $\epsilon$. With $\epsilon \approx 1 a_{0} / \mathrm{eV}$ a realistic quantum yield is obtained and the main part of the energy distribution is in satisfactory agreement with experimental results.

\section{Influence of frustrated hops}

"Frustrated hops" occur when a surface hop is abandoned because adjustment of the kinetic energy to conserve total energy is not possible. Only upward hops can be frustrated. To determine the effect of our simple method of conserving total energy at hops, we examined carefully a representative set of 10000 two-state trajectories at total energy $5 \mathrm{eV}$. In this group, 747 trajectories finished on the R-state PES for a triplet yield of $7.47 \%$. In 167 trajectories, the last hop attempt was frustrated. Of those, 76 finished on B and 91 finished on R. We conclude that frustrated hops may cause an overestimation of the triplet yield of the order of $0.2 \%$. This bias is smaller than the other theoretical uncertainties and also the experimental uncertainty. In addition, it is probably partially compensated for in our empirical selection of the coupling width parameter $\epsilon$, since larger values of $\epsilon$ lead to slightly higher triplet yields.

\section{Wavelength dependence of quantum yields}

The wavelength dependence of the quantum yield $\Gamma\left({ }^{1} D\right)$ for the singlet channel is depicted in Fig. 15(a). It steeply rises from zero at the classical threshold for channel (2) (322 $\mathrm{nm}$ for the PESs used in these calculations), reaches a maximum of nearly 1.0 between 295 and $300 \mathrm{~nm}$, and then gradually decreases to shorter wavelengths. In order to assess the accuracy of the TSH approximation, we also show the quantum mechanical $\Gamma\left({ }^{1} D\right)$ obtained from two-state wave packet calculations ${ }^{54}$ similar to those described by Grebenshchikov et al. ${ }^{10}$ The same diabatic potentials and the same coupling potential $V_{\mathrm{BR}}$ are employed in the classical and the quantum mechanical calculations. The quantum mechanical $\Gamma\left({ }^{1} D\right)$ exhibits pronounced resonance structures whose intensities become larger with increasing wavelength, toward the quantum mechanical threshold of channel (2) at $314.9 \mathrm{~nm}$. These structures, like the structures in the quantum mechanical absorption spectrum, are due to short-lived resonances high in the continuum of the two $C_{s}$ wells of the B-state PES. ${ }^{10}$ The widths are naturally smallest near the threshold which explains why the structures are most pronounced at the onset of the Hartley band $(>280 \mathrm{~nm})$. The classical and quantum mechanical quantum yields agree quite well over the entire range of wavelengths except for the
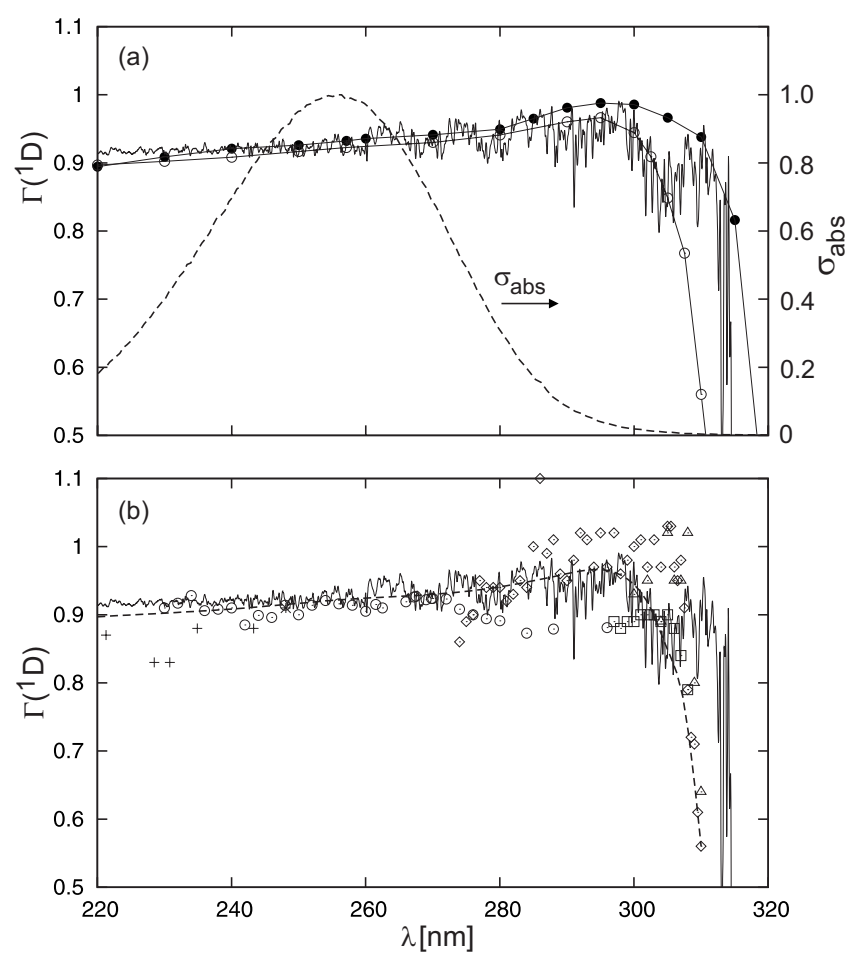

FIG. 15. (a) Singlet channel quantum yield $\Gamma\left({ }^{1} D\right)$ as function of wavelength. Comparison of quantum mechanical [solid line, courtesy of Grebenshchikov (Ref. 54)] and original $(\bullet)$ and zero-point energy corrected $(\bigcirc$ ○) TSH results. In order to relate $\Gamma\left({ }^{1} D\right)$ to the absorption spectrum, the absorption cross section $\sigma_{\text {abs }}$ calculated by the classical approach is also shown (right-hand scale, arbitrary units). (b) Comparison of the quantum mechanical and the corrected TSH (dashed line) $\Gamma\left({ }^{1} D\right)$ with experimental data: (○) Takahashi et al. (Ref. 53), ( $\square$ ) Taniguchi et al. (Ref. 62), (+) Cooper et al. (Ref. 60), $(\diamond)$ Trolier and Wiesenfeld (Ref. 66), and $(\triangle)$ Ball et al. (Ref. 63). For clarity the experimental error bars are not shown; they are typically of the order of $0.03-0.05$.

expected absence of resonance structures in the semiclassical calculation and the disagreement near threshold. The agreement is particularly good near the maximum of the absorption spectrum and less favorable where the absorption cross section is small $(\lambda \gtrsim 285 \mathrm{~nm})$, i.e., where a classical description of the photodissociation is in general most uncertain. ${ }^{30}$

The agreement of the classical with the quantum mechanical $\Gamma\left({ }^{1} D\right)$ can be further improved, especially in the threshold region, by incorporating in a very simple way the zero-point energy (ZPE) of $\mathrm{O}_{2}\left({ }^{1} \Delta_{g}\right)$. This is achieved by adding a potential term $E_{\mathrm{ZP}} f\left(R_{1}\right)$ to the B-state PES, where $R_{1}>R_{2}$ is the dissociation bond, $E_{\mathrm{ZP}}=0.091 \mathrm{eV}$ is the zeropoint energy, and $f\left(R_{1}\right)$ is a switching function. We used $f$ $=0$ or 1 for $R_{1}<4 a_{0}$ or $R_{1}>6 a_{0}$, respectively, and $f\left(R_{1}\right)$ $=\sin ^{2}\left[\pi\left(R_{1}-4\right) / 4\right]$ for $4 \leq R_{1} \leq 6$. Raising the classical threshold increases, for the same total energy, the lifetime of the trajectories which, in turn, enhances the probability for hopping to the R-state PES and thereby diminishes the singlet quantum yield. The effect is very small at the shortest wavelengths $(\lambda \lesssim 220 \mathrm{~nm})$, and of the order of $1 \%$ for the main part of the Hartley band, but becomes dramatic near the threshold [Fig. 15(a)]. The agreement between the quantum and ZPE-corrected TSH yields is excellent except for the slight maximum around $310 \mathrm{~nm}$ in the quantum mechanical yield, very close to the threshold. 


\section{Lifetime in Huggins band}

The Huggins band is due to excitation of the vibrational states supported by the two $C_{s}$ wells of the B-state PES. ${ }^{12,55}$ Below the threshold for channel (2) the lifetimes of these states are governed by coupling to dissociative states, primarily the spin-allowed transition to the $\mathrm{R}$ state, which intersects the B-state PES close to its minimum (Fig. 6). Predissociation due to coupling to triplet states is presumably inefficient. Comparison of calculated lifetimes with measured ones is thus another test of the B/R coupling strength.

Within the TSH approach we determine survival times with the following procedure. (a) Trajectories with a specific total energy are started in one of the wells with arbitrary initial conditions. (b) They are first run for 1 ps on the B-state PES with $V_{\mathrm{BR}}$ set to zero, in order to allow for energy exchange between all degrees of freedom. (c) After this initialization time the coupling is switched on and the trajectory is followed until dissociation in the R-state. (d) The time between switching $V_{\mathrm{BR}}$ on and the hop to the R-state PES is defined as survival time $\tau$. The distribution of $\tau$ cannot be well represented by a single exponential and the extraction of a lifetime is somewhat uncertain. We therefore determine the average survival time, $\tau_{\mathrm{av}}$, by averaging over all trajectories. Calculations are performed for $E=3.6 \mathrm{eV}(262 \mathrm{~nm}), 3.8 \mathrm{eV}$ $(342 \mathrm{~nm})$, and $4.0 \mathrm{eV}(324 \mathrm{~nm})$; the numbers in brackets are the corresponding excitation wavelengths. For each energy 50000 trajectories are run. The average survival times, for increasing energy, are $1.6,1.2$, and 0.8 ps. With increasing energy the molecule stretches to gradually longer $\mathrm{O}_{2}$ bond lengths. This increases the coupling potential $V_{\mathrm{BR}}$ (Fig. 9) and thus reduces the survival time. Furthermore, a singleexponential description becomes gradually better with increasing energy. $\tau_{\mathrm{av}}$ does not depend on the initialization time; a second calculation at $3.8 \mathrm{eV}$ using a 3 ps initialization time gave the same results.

The vibrational lines of the Huggins band are substantially broadened by temperature. ${ }^{56,57}$ Therefore, in order to obtain trustworthy information about the lifetime one has to work with very cold ozone. Sinha et al. ${ }^{58}$ took excitation spectra of rotationally cooled $\mathrm{O}_{3}\left(T_{\text {rot }} \sim 3 \mathrm{~K}\right)$ in the region of $325 \mathrm{~nm}$ and observed partly resolved rotational structures. From the contour analysis they derived an upper bound of $3.6 \mathrm{ps}$ for vibrational state $(6,0,0)$. The survival times obtained by the TSH approach are compatible with this estimate. Decreasing the coupling by a factor of 0.5 yields for $E=3.8 \mathrm{eV}$ a survival time of $2.6 \mathrm{ps}$, slightly more than double the value for the unscaled $V_{\mathrm{BR}}$.

\section{B. Four-state calculations: $B, R, A$, and $X$}

TSH calculations are also performed for four states: B, $\mathrm{A}, \mathrm{R}$, and $\mathrm{X}$. In these calculations couplings between states $\mathrm{B}$ and $\mathrm{R}, \mathrm{B}$ and $\mathrm{A}, \mathrm{A}$ and $\mathrm{X}$, and $\mathrm{A}$ and $\mathrm{R}$ are considered, while $\mathrm{B} / \mathrm{X}$ and $\mathrm{R} / \mathrm{X}$ couplings are neglected. The $\mathrm{A} / \mathrm{X}$ and $\mathrm{A} / \mathrm{R}$ couplings are given by Eq. (9) with $\Delta E_{x}=0.05$ and $0.04 \mathrm{eV}$, respectively, with $\kappa=0.25 \mathrm{eV}$ for both coupling potentials. Calculations are performed for $E=5 \mathrm{eV}$ and several values of $\epsilon$ for $V_{\mathrm{BR}}$; in all cases $\epsilon=1 a_{0} / \mathrm{eV}$ for $V_{\mathrm{BA}}$. The results for the triplet yield are compared with the results of the two- state calculations in Fig. 13. The deviations are of the same order as the statistical uncertainty, i.e., allowing transitions from state B to the other states does not change the quantum yield for the triplet channel. Transitions from B to A are rare; the probability to reach the singlet channel via the A-state is only $0.4 \%-0.7 \%$. Therefore it is not surprising that the probability for finishing in channel (1) via the $\mathrm{B} \rightarrow \mathrm{A} \rightarrow \mathrm{X}$ pathway is essentially zero. The probability for two-step transitions $\mathrm{B} \rightarrow \mathrm{A} \rightarrow \mathrm{R}$ has not been explicitly calculated, because the triplet yield is dominated by the transitions $\mathrm{B} \rightarrow \mathrm{R}$. However, it is also expected to be negligible.

The probability for accessing state A does increase with $\epsilon$ in the expression for $V_{\mathrm{BA}}$; for $\epsilon=4 a_{0} / \mathrm{eV}$, for example, it is of the order of $2 \%$. The probability for dissociation via the ground state $X$ also slightly increases, but even for $\epsilon=4$ it is only about $0.1 \%$. Increasing $V_{\mathrm{BA}}$ by an overall factor of 2 doubles the probability to access $\mathrm{A}$ and also increases the probability to dissociate via $X$ by a factor $2-4$, but does not noticeably change the triplet yield.

\section{DISCUSSION}

The structure of the electronic states (possibly) involved in the UV photodissociation via the Huggins/Hartley band system, i.e., the lower ${ }^{1} A^{\prime}$ states, is intricate and characterized by several avoided crossings. All states are interconnected, either directly or indirectly via two-step transitions like $\mathrm{B} \rightarrow \mathrm{A} \rightarrow \mathrm{X}$. Avoided crossings between two states usually occur in different regions of the three-dimensional coordinate space. However, three states sometimes strongly interact in the same coordinate region. The density of states increases and the coupling behavior becomes more complicated with increasing excitation energy. This is especially the case towards the breakup into three $\mathrm{O}\left({ }^{3} P\right)$ atoms when many states coalesce; the threshold for the $3 \mathrm{O}\left({ }^{3} P\right)$ channel lies in the high-energy tail of the Hartley band (198 nm). The energetic separation of two interacting states at an avoided crossing varies with coordinates: While in one region of coordinate space the adiabatic splittings may be small and the nuclear motion essentially diabatic, in another region the splittings may be larger and the dynamics more adiabatic.

All these circumstances make the construction of diabatic_-diagonal and off-diagonal_potentials for dynamics calculations difficult. A rigorous diabatization as applied for the two lowest ${ }^{1} A$ " states (Chappuis band) or the lowest two ${ }^{3} A^{\prime \prime}$ states (Wulf band) ${ }^{10}$ does not appear feasible. The "diabatization by eye" adopted in the present study is certainly not the ultimate approach. The uncertainties in the shape of the diabatic PESs are largest when more than two states interact in the same region of coordinate space or when the spacing near an avoided crossing is large. For the two most important states, $\mathrm{B}$ and $\mathrm{R}$, the latter occurs predominantly when both $\mathrm{O}-\mathrm{O}$ bonds $R_{1}$ and $R_{2}$ are significantly stretched and the bond angle $\alpha$ is relatively small $\left(\$ 90^{\circ}\right)$. The off-diagonal potential matrix elements, which are essentially proportional to the splittings of the adiabatic curves at the avoided crossings, suffer from the same uncertainties, i.e., they are less well defined in regions where the adiabatic splittings are largest. In spite of these limitations, the diabatic 
PESs and coupling potentials constructed here enable us to study the photodissociation of ozone in the Hartley band in an unprecedented manner.

The dynamics calculations presented in this study are performed in the semiclassical TSH approach, which is a combination of a quantum mechanical description of the electronic motion and a classical treatment of the heavy particle dynamics. The comparison of the TSH triplet/singlet quantum yields with exact wave packet calculations establishes that the TSH approach is trustworthy for the main part of the Hartley band, especially when the zero-point energy in the singlet channel is approximately incorporated [Fig. 15(a)]. The agreement is perfect-except for the quantum mechanical resonance structures-around the maximum of the band; in the short-wavelength tail the TSH triplet yield is merely $10 \%$ larger than the quantum mechanical one. The deviations are largest at the onset of the Hartley band, close to the threshold of the singlet channel. This is not unexpected because classical mechanics is usually less accurate near a threshold, where the kinetic energy is almost zero. The generally good performance of the TSH approximation has two origins: the high excess energy on the B-state PES and the rapid fragmentation on the R-state PES once a transition has been made. Both guarantee that the main part of the dissociation is fast and direct, conditions for which classical mechanics is generally trustworthy. ${ }^{30}$

The trajectory calculations provide easy access to the distribution of transition locations. In Fig. 16 we show for three wavelengths the distribution $P_{\text {cross }}\left(R_{2}\right)$ of the product $\mathrm{O}_{2}$ bond length when a trajectory traverses for the first time the $\mathrm{B} / \mathrm{R}$ crossing seam, whether a transition occurred or not. $P_{\text {cross }}\left(R_{2}\right)$ for the largest $\lambda$ peaks around $R_{2} \approx 2.3 a_{0}$ and then rapidly decays to zero around $2.5 a_{0}$; for this wavelength the trajectories on the B-state PES have little excitation in the $\mathrm{O}_{2}$ vibrational coordinate. As the wavelength shortens, i.e., the total energy increases, the peak shifts to longer $\mathrm{O}_{2}$ bond lengths and at the same time the distribution becomes significantly broader; $P_{\text {cross }}\left(R_{2}\right)$ for $220 \mathrm{~nm}$ extends to $R_{2}$ $=3.4 a_{0}$. Also shown are the distributions $P_{\text {hop }}\left(R_{2}\right)$ of $R_{2}$ when the last hop from the B-state PES to the R-state PES occurred. They indicate where the $\mathrm{B} \rightarrow \mathrm{R}$ transitions take place. In view of the dependence of $V_{\mathrm{BR}}$ on $R_{2}$ (Fig. 9) and the increase in the potential energy as the $\mathrm{O}_{2}$ bond is stretched, it is not surprising that $P_{\text {hop }}\left(R_{2}\right)$ increases with $R_{2}$, reaches a broad maximum, and then diminishes. The shape of $P_{\text {hop }}\left(R_{2}\right)$ determines the kinetic energy distribution in the triplet channel. ${ }^{20}$ The transition probability defined as $P_{\text {trans }}$ $=P_{\text {hop }} / P_{\text {cross }}$ specifies the percentage of trajectories in each $R_{2}$ interval that undergo a transition to the R-state. An example is shown in Fig. 16(e). It strongly peaks at large $R_{2}$ where the coupling element is largest and at the same time the residence time is longest, i.e., the kinetic energy is smallest. $P_{\text {trans }}$ is well approximated by the Landau-Zener transition probability computed as a function of $R_{2}$.

Figure 16(d) shows the crossing and hopping distributions as functions of the bond angle $\alpha$ for $250 \mathrm{~nm}$; both are quite narrow. $P_{\text {cross }}(\alpha)$ peaks around $100^{\circ}$, while $P_{\text {hop }}(\alpha)$ has its maximum around $93^{\circ}$. As for the $R_{2}$-dependence, the transition probability $P_{\text {trans }}=P_{\text {hop }} / P_{\text {cross }}$ (not shown) is largest
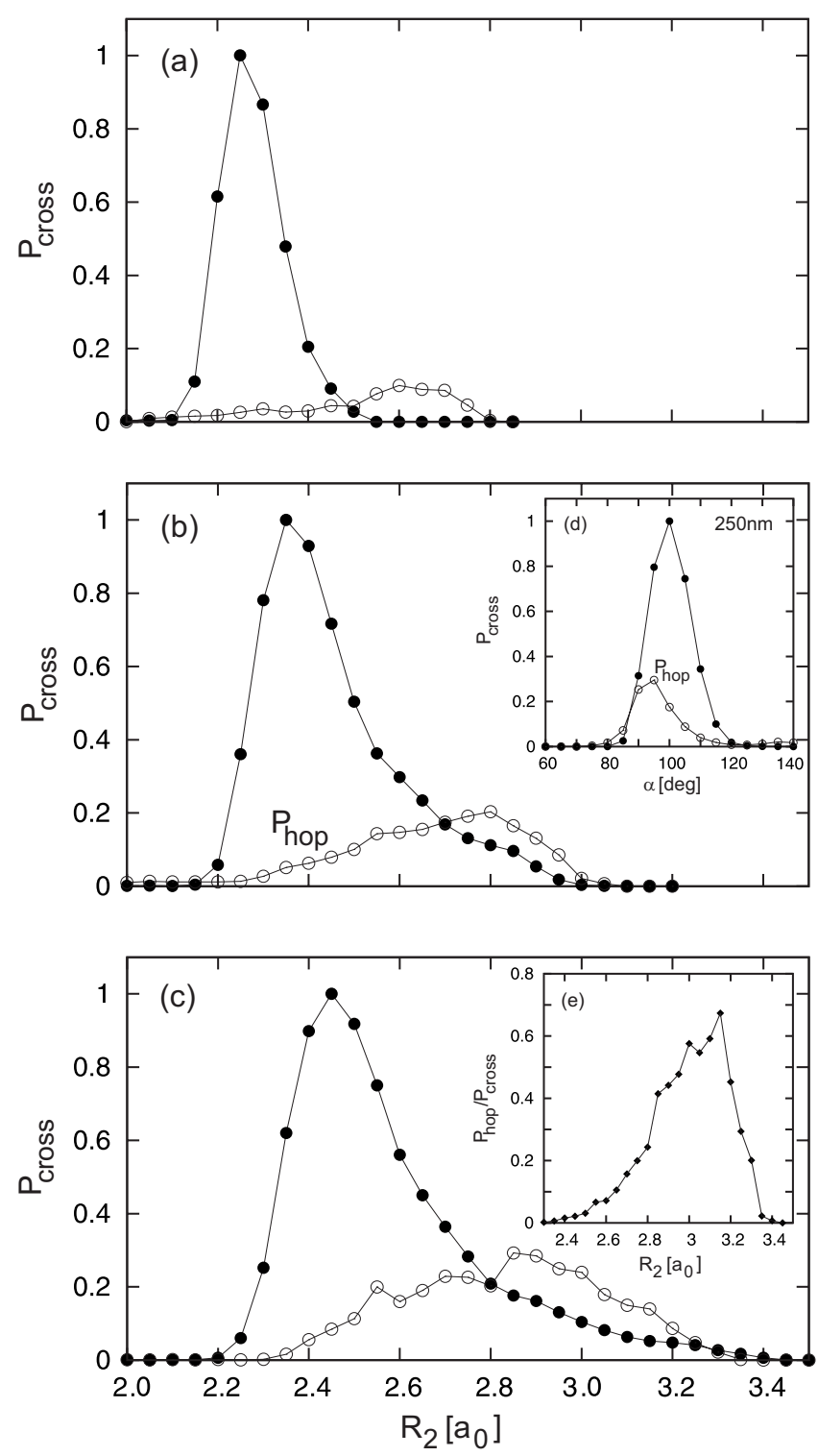

FIG. 16. Crossing probability $P_{\text {cross }}(\bullet)$ and hopping probability $P_{\text {hop }}(\bigcirc$ O) vs $\mathrm{O}_{2}$ bond length $R_{2}$ for $\lambda=280 \mathrm{~nm}$ (a), $250 \mathrm{~nm}$ (b), and $220 \mathrm{~nm}$ (c). For each wavelength $P_{\text {cross }}$ is normalized to one; the hopping probability for each $\lambda$ is multiplied by the same normalization constant and, for clarity, additionally multiplied by 4 . (d) $P_{\text {cross }}$ and $P_{\text {hop }}$ vs bond angle $\alpha$ for $\lambda$ $=250 \mathrm{~nm}$. (e) The transition probability $P_{\text {hop }} / P_{\text {cross }}$ for $220 \mathrm{~nm}$. See text for more details.

where the coupling matrix element is largest and the kinetic energy is smallest. Angles of $80^{\circ}-90^{\circ}$ mark the first turning point of the trajectories, when they are deflected at the high barrier separating the equivalent potential wells. We emphasize that the transitions from $\mathrm{B}$ to $\mathrm{R}$ often occur in regions of coordinate space where the diabatization and the construction of the coupling element are ambiguous because several adiabatic states are close in energy.

The $\mathrm{O}\left({ }^{1} D\right)$ quantum yield as a function of wavelength is a very important quantity in atmospheric chemistry and therefore has been measured in a number of laboratories. ${ }^{2,53,59-67}$ In Fig. 15(b) we compare the quantum mechanical and the TSH $\Gamma\left({ }^{1} D\right)$ with measured data. According to the experiments of Takahashi et al. ${ }^{53}$ and Taniguchi et al. ${ }^{62} \Gamma\left({ }^{1} D\right)$ is almost constant, $0.87 \pm 0.02-0.93 \pm 0.02$, in 
the range $230 \mathrm{~nm} \leq \lambda \leq 305 \mathrm{~nm}$. Earlier experiments by Trolier and Wiesenfeld ${ }^{66}$ yielded values between $\sim 0.9$ and $\sim 1.0$ for $275 \mathrm{~nm} \leq \lambda \leq 305 \mathrm{~nm}$ with a broad maximum between 285 and $305 \mathrm{~nm}$. Ball et al. ${ }^{63}$ measured values between $0.89 \pm 0.05$ and $1.0 \pm 0.05$ from 300 to $308 \mathrm{~nm}$. For wavelengths above $\sim 305 \mathrm{~nm}$ all measured ${ }^{1} D$ quantum yields rapidly decrease. Both the quantum mechanical and the TSH $\Gamma\left({ }^{1} D\right)$ gradually rise from a value of about 0.90 0.91 at $220 \mathrm{~nm}$ to $0.95-0.97$ around $300 \mathrm{~nm}$ and then rapidly drop to zero toward the threshold for channel (2). Thus, the theoretical predictions are in satisfactory accord with the experimental data from 220 through $310 \mathrm{~nm}$, with the reservation that the experimental data show substantial scatter for $275 \mathrm{~nm}<\lambda<310 \mathrm{~nm}$. A comparison is unreasonable for wavelengths $\gtrsim 310 \mathrm{~nm}$ because, first, the theoretical threshold for channel (2) is lower and, second, dissociation via hot vibrational bands, not taken into account in the present study, becomes important. ${ }^{2}$ In the short-wavelength tail of the Hartley band the experimental $\Gamma\left({ }^{1} D\right)$ rapidly decays from about 0.90 at $220 \mathrm{~nm}$ to about 0.5 at $193 \mathrm{~nm},{ }^{67}$ while the TSH $\Gamma\left({ }^{1} D\right)$ decays only slightly $(0.87$ at $200 \mathrm{~nm})$. However, with decreasing wavelength higher electronic states become excited that are not considered in the present study. At $193 \mathrm{~nm}$, for example, the dissociation occurs primarily via states $5^{1} A^{\prime}$ and $4{ }^{1} A^{\prime \prime}$ rather than the B-state $\left(3{ }^{1} A^{\prime}\right) .{ }^{27}$

The satisfactory agreement with the experimental data suggests that the $\mathrm{B} / \mathrm{R}$ off-diagonal potential used in the calculations is realistic. However, one has to realize that $\Gamma\left({ }^{1} D\right)$ is not very sensitive to $V_{\mathrm{BR}}$. Multiplication of $V_{\mathrm{BR}}$ by an overall factor of $0.75-1.5$ would still yield an acceptable agreement for the main part of the Hartley band (Fig. 14). The quantum yield is even less sensitive to the width parameter $\epsilon$, the only free parameter of the entire calculation. Near the maximum of the band values of $\epsilon=0.5$ through $4 a_{0} / \mathrm{eV}$ would be compatible with the experimental data (Fig. 13).

Brouard et $a l .{ }^{49}$ measured the distribution of spin-orbit states for the very slow products in channel (1) and found it to be markedly nonstatistical, with the lowest state ${ }^{3} P_{2}$ of the $\mathrm{O}$ atom being preferentially produced. That observation led them to the suggestion that these slow products are created by dissociation via the ground state $\mathrm{X}$ rather than the repulsive state R; according to the work of Rosmus et al. ${ }^{68}$ and Tashiro and Schinke ${ }^{69}$ the ground state of ozone adiabatically correlates with $\mathrm{O}\left({ }^{3} P_{2}\right)$ while the R-state connects with $\mathrm{O}\left({ }^{3} P_{0}\right)$. Brouard et $a l^{49}$ therefore proposed a dissociation mechanism for the production of the very slow products consisting of the following steps: (a) excitation of the B-state, (b) transition to the A-state, (c) nuclear motion into the region of cyclic ozone, (d) transition to the X-state near the $\mathrm{A} / \mathrm{X}$ crossing seam, and (e) final dissociation on the lowest PES. This appealing scenario motivated the present study.

TSH calculations at $226 \mathrm{~nm}$ including all four states force us to abandon this concept. The probability for dissociation on the A-state is only about half a percent and the probability for accessing the triplet channel via the X-state is practically zero-four trajectories out of 200 000. Most trajectories that reach the A-state dissociate in the singlet channel. The excess energy with respect to channel (2) at $226 \mathrm{~nm}$ is high, about $1.6 \mathrm{eV}$, and therefore the survival time in the cyclic well is short. Of course, by changing the parameters in the coupling potentials $V_{\mathrm{BA}}$ and/or $V_{\mathrm{AX}}$ it is possible to artificially increase the quantum yield in the $\mathrm{X}$-state. For example, increasing $\overline{\Delta E}_{x}$ in Eq. (9) for $V_{\mathrm{AX}}$ by a factor of 10 yields $0.03 \%$ of all trajectories ending in state $\mathrm{X}$. Enlarging additionally $V_{\mathrm{BA}}$ by a factor of 10 gives a quantum yield of $0.6 \%$ for $\mathrm{X}$. However, such large variations are not justified by the electronic structure calculations.

The few trajectories that eventually hop to the X-state do this over a wide range of $\mathrm{O}_{2}$ bond distances $R_{2}$ (Fig. 7) with the consequence that the translational energy distribution extends rather uniformly from small to large values. This is true for the original coupling potentials as well as those which are artificially increased. In order to yield a prominent peak at very small $E_{\text {tr }}$, a majority of trajectories would have to hop from A to $\mathrm{X}$ at $R_{2}>3.5 a_{0}$ and $R_{1}>4.5 a_{0}$ when the repulsion between $\mathrm{O}$ and $\mathrm{O}_{2}$ on the ground-state PES is almost zero and, therefore, the extremely high energy stored in $\mathrm{O}_{2}$ vibration at the hop to the $\mathrm{X}$-state PES remains there. This appears implausible.

The A-state can, in principle, also be accessed by direct photoexcitation from the ground state. However, $\mu_{\mathrm{XA}}$ is very small. ${ }^{11,70}$ The value around the FC point $\left(2.43 a_{0}, 117^{\circ}\right)$ is 0.0071 a.u. and the ratio $\left(\mu_{\mathrm{XA}} / \mu_{\mathrm{XB}}\right)^{2}$ is of the order of 7 $\times 10^{-5}$. We calculated the $\mathrm{X} \rightarrow \mathrm{A}$ absorption cross section within the classical approximation with constant $\mu_{\mathrm{XA}}$, where the constant is set to the value at the FC geometry. It has a maximum at $\sim 300 \mathrm{~nm}$, compared to $256 \mathrm{~nm}$ for the B-state. At $226 \mathrm{~nm}$ the A-state absorption cross section is about $10^{-6}$ times smaller than the B-state cross section. This eliminates direct absorption into the A-state as a realistic explanation of the peak at very small translational energies in the triplet channel.

Recent experiments and accompanying electronic structure calculations provided convincing evidence that the narrow peaks in the triplet channel translational energy distribution near $E_{\mathrm{tr}} \approx 0$ for $\lambda=226 \mathrm{~nm}$ are due to the excitation of the excited triplet (Herzberg) states. ${ }^{14}$ The branching ratios for the singlet and the Herzberg channels are determined by the nonadiabatic couplings when the product $\mathrm{O}_{2}$ swings through the critical region $R_{2} \approx 2.8 a_{0}$ as $\mathrm{O}$ and $\mathrm{O}_{2}$ separate (Fig. 5). The nonadiabatic coupling is effective only at intermediate $\mathrm{O}-\mathrm{O}_{2}$ separations. It gradually diminishes with increasing $\mathrm{O}-\mathrm{O}_{2}$ separations and therefore a small fraction of the $\mathrm{O}_{2}$ fragments is trapped in the Herzberg states. Because many states interact in this part of the coordinate space, especially when spin-orbit coupling is taken into account, quantitative assessments are not currently possible. Nevertheless, it is plausible to assume that preferentially the lower electronic states are populated while $\mathrm{O}$ and $\mathrm{O}_{2}$ separate, and they naturally correlate with $\mathrm{O}\left({ }^{3} P_{2}\right)$. This would, at least qualitatively, explain the observation made by Brouard et al. ${ }^{49}$ More detailed discussions of the absorption spectrum, especially the role of thermal broadening, and the product energy distributions both in the singlet and the triplet channels will be published in forthcoming articles. 


\section{SUMMARY}

We calculated the potential energies of the first five electronic states of ozone with ${ }^{1} A^{\prime}$ symmetry employing the MRD-CI level of theory and the aug-cc-pVTZ atomic basis set. All internal coordinates- the two $\mathrm{O}-\mathrm{O}$ bond lengths $\left(R_{1}\right.$ and $\left.R_{2}\right)$ and the bond angle $(\alpha)$ - have been varied over wide ranges. These first five states exhibit several avoided crossings. The splittings at the avoided crossings depend appreciably on the coordinates with the consequence that in some regions of coordinate space an adiabatic representation is appropriate, while in other regions a diabatic representation is needed. Smooth diabatic PESs have been constructed for the four lowest states (X, A, B, and R) by selecting energies that apparently belong to the same diabatic state and interpolating these energies by three-dimensional cubic splines. These PESs are more accurate than those published before ${ }^{9}$ and are a good basis for multistate dynamics calculations.

Off-diagonal coupling matrix elements- $V_{\mathrm{BR}}, V_{\mathrm{BA}}, V_{\mathrm{AX}}$, and $V_{\mathrm{AR}}$-have also been constructed from supplementary electronic structure calculations on narrower coordinate grids than those used to construct the PESs. The largest effort has been devoted to the construction of the coupling element $V_{\mathrm{BR}}$, which governs the important transition from the originally excited state $\mathrm{B}$ to the repulsive state $\mathrm{R}$ and therefore mainly determines the singlet/triplet branching ratio. In accordance with general diabatization procedures,${ }^{30}$ all coupling elements are proportional to half of the energy separation of the respective adiabatic potentials at the avoided crossings, $\Delta E_{x} / 2$, and this quantity is obtained from electronic structure calculations.

The semiclassical TSH method of Tully ${ }^{52}$ has been employed to investigate the dissociation dynamics including nonadiabatic transitions. In most of the TSH calculations only states B and R have been included. Except for resonance structures and the near-threshold region, the semiclassical quantum yield for the singlet channel agrees well with the results of quantum mechanical wave packet calculations performed with the same PESs and coupling matrix elements. ${ }^{54}$ The calculated quantum yields agree well with experimental results over the entire range of the Hartley absorption band.

TSH calculations including all four states have shown that transitions from state B to state A, which also connects with the singlet channel, do not play an important role; for example, their probabilities are below $1 \%$ for $\lambda=226 \mathrm{~nm}$. Since the coupling between the A-state and the $\mathrm{X}$ - and $\mathrm{R}$-states is weak, pathways $\mathrm{B} \rightarrow \mathrm{A} \rightarrow \mathrm{X}$ and $\mathrm{B} \rightarrow \mathrm{A} \rightarrow \mathrm{R}$ are negligible. The two-state model suffices to describe the main part of the photodissociation of ozone in the Hartley band. We conclude that the dissociation mechanism suggested in Ref. 49 is unable to explain the very slow $\mathrm{O}\left({ }^{3} P\right)$ products in the triplet channel.

\section{ACKNOWLEDGMENTS}

Financial support by the Deutsche Forschungsgemeinschaft and the Fonds der Chemischen Industrie is gratefully acknowledged. The authors are grateful to S. Yu. Grebenshchikov for many stimulating discussions and the permission to use the quantum mechanical quantum yield prior to publication, and to L. T. Nguyen for work on the TSH calculations and their interpretation.

${ }^{1}$ H. S. Johnston, Annu. Rev. Phys. Chem. 43, 1 (1992).

${ }^{2}$ Y. Matsumi and M. Kawasaki, Chem. Rev. (Washington, D.C.) 103, 4767 (2003)

${ }^{3}$ P. L. Houston, in Modern Trends in Chemical Reaction Dynamics: Experiment and Theory (Part 2), Advanced Series in Physical Chemistry Vol. 14, edited by X. Yang and K. Liu (World Scientific, Singapore, 2004).

${ }^{4}$ D. E. Freeman, K. Yoshino, J. R. Esmond, and W. H. Parkinson, Planet. Space Sci. 32, 239 (1984).

${ }^{5}$ C. Leforestier, F. Le Quéré, K. Yamashita, and K. Morokuma, J. Chem. Phys. 101, 3806 (1994).

${ }^{6}$ N. Balakrishnan and G. D. Billing, J. Chem. Phys. 101, 2968 (1994).

${ }^{7}$ M. Alacid and C. Leforestier, J. Chem. Phys. 114, 1685 (2001).

${ }^{8}$ E. Baloïtcha and G. G. Balint-Kurti, J. Chem. Phys. 123, 014306 (2005).

${ }^{9}$ Z.-W. Qu, H. Zhu, S. Yu. Grebenshchikov, and R. Schinke, J. Chem. Phys. 123, 074305 (2005).

${ }^{10}$ S. Yu. Grebenshchikov, Z.-W. Qu, H. Zhu, and R. Schinke, Phys. Chem. Chem. Phys. 9, 2044 (2007).

${ }^{11}$ Z.-W. Qu, H. Zhu, and R. Schinke, Chem. Phys. Lett. 377, 359 (2003).

${ }^{12}$ Z.-W. Qu, H. Zhu, S. Yu. Grebenshchikov, R. Schinke, and S. C. Farantos, J. Chem. Phys. 121, 11731 (2004)

${ }^{13}$ P. J. Hay, R. T. Pack, R. B. Walker, and E. J. Heller, J. Phys. Chem. 86, 862 (1982).

${ }^{14}$ R. Schinke, G. C. McBane, L. Shen, P. C. Singh, and A. G. Suits, J. Chem. Phys. 131, 011101 (2009).

${ }^{15}$ K. Yamashita, K. Morokuma, F. Le Quéré, and C. Leforestier, Chem. Phys. Lett. 191, 515 (1992).

${ }^{16}$ G. Barinovs, N. Marković, and G. Nyman, Chem. Phys. Lett. 315, 282 (1999).

${ }^{17}$ S. Y. Lin, K. L. Han, and G. Z. He, J. Chem. Phys. 114, 10651 (2001).

${ }^{18}$ S. Yu. Grebenshchikov and R. Schinke, J. Chem. Phys. 126, 247101 (2007).

${ }^{19}$ G. G. Balint-Kurti and E. Baloïtcha, J. Chem. Phys. 126, 247102 (2007).

${ }^{20}$ Z.-W. Qu, H. Zhu, S. Yu. Grebenshchikov, and R. Schinke, J. Chem. Phys. 122, 191102 (2005).

${ }^{21}$ H. Zhu, Z.-W. Qu, M. Tashiro, and R. Schinke, Chem. Phys. Lett. 384, 45 (2004).

${ }^{22}$ H.-J. Werner and P. J. Knowles, J. Chem. Phys. 89, 5803 (1988).

${ }^{23}$ P. J. Knowles and H.-J. Werner, Chem. Phys. Lett. 145, 514 (1988).

${ }^{24}$ S. R. Langhoff and E. R. Davidson, Int. J. Quantum Chem. 8, 61 (1974).

${ }^{25}$ T. H. Dunning, Jr., J. Chem. Phys. 90, 1007 (1989).

${ }^{26}$ MOLPRO, a package of $a b$ initio programs written by H.-J. Werner and P. J. Knowles, Version 2006.1, R. Lindh, F. R. Manby, M. Schütz, et al.

${ }^{27}$ R. Schinke and S. Yu. Grebenshchikov, Chem. Phys. 347, 279 (2008).

${ }^{28}$ R. P. Saxon and B. Liu, J. Chem. Phys. 67, 5432 (1977).

${ }^{29}$ T. G. Slanger and P. C. Cosby, J. Phys. Chem. 92, 267 (1988).

${ }^{30}$ R. Schinke, Photodissociation Dynamics (Cambridge University Press, Cambridge, 1993)

${ }^{31}$ S. Yu. Grebenshchikov, R. Schinke, Z.-W. Qu, and H. Zhu, J. Chem. Phys. 124, 204313 (2006).

${ }^{32}$ The double-well behavior of the B-state PES was also apparent in the calculations of Baloïtcha and Balint-Kurti (Fig. 1 of Ref. 8). It had also been seen by Qu et al. (Ref. 9), but had been erased by their analytical interpolation.

${ }^{33}$ R. Schinke, S. Yu. Grebenshchikov, M. V. Ivanov, and P. Fleurat-Lessard, Annu. Rev. Phys. Chem. 57, 625 (2006).

${ }^{34}$ In the more rigorous diabatization performed for the two lowest ${ }^{1} A$ " states responsible for the Chappuis band of ozone (Ref. 31), for example, the coupling element $V_{12}$ has a large constant value in the dissociation channel. On the other hand, the two diabatic potentials become equal at large $\mathrm{O}-\mathrm{O}_{2}$ separations and their offsets from the adiabatic potentials are $+V_{12}$ and $-V_{12}$, respectively. Thus, the transformation from the diabatic to the adiabatic representation would recover the original adiabatic potentials.

${ }^{35}$ P. J. Hay, T. H. Dunning, Jr., and W. A. Goddard III, J. Chem. Phys. 62, 3912 (1975).

${ }^{36}$ P. J. Hay and T. H. Dunning, Jr., J. Chem. Phys. 67, 2290 (1977).

${ }^{37}$ S. S. Xantheas, G. J. Atchity, S. T. Elbert, and K. Ruedenberg, J. Chem. Phys. 94, 8054 (1991). 
${ }^{38}$ A. Banichevich and S. D. Peyerimhoff, Chem. Phys. 174, 93 (1993).

${ }^{39}$ G. J. Atchity and K. Ruedenberg, Theor. Chem. Acc. 96, 176 (1997).

${ }^{40}$ J. Ivanic, G. J. Atchity, and K. Ruedenberg, J. Chem. Phys. 107, 4307 (1997).

${ }^{41}$ R. Siebert, P. Fleurat-Lessard, R. Schinke, M. Bittererová, and S. C. Farantos, J. Chem. Phys. 116, 9749 (2002).

${ }^{42}$ A. Kalemos and A. Mavridis, J. Chem. Phys. 129, 054312 (2008).

${ }^{43}$ F. Grein, J. Chem. Phys. 130, 124118 (2009).

${ }^{44}$ S. S. Xantheas, S. T. Elbert, and K. Ruedenberg, J. Chem. Phys. 93, 7519 (1990).

${ }^{45}$ G. J. Atchity and K. Ruedenberg, J. Chem. Phys. 99, 3790 (1993).

${ }^{46}$ G. J. Atchity, K. Ruedenberg, and A. Nanayakkara, Theor. Chem. Acc. 96, 195 (1997).

${ }^{47}$ G. J. Atchity and K. Ruedenberg, Theor. Chem. Acc. 97, 47 (1997).

${ }^{48}$ N. Sukumar and S. D. Peyerimhoff, Mol. Phys. 95, 61 (1998).

${ }^{49}$ M. Brouard, A. Goman, S. J. Horrocks, A. J. Johnsen, F. Quadrini, and W.-H. Yuen, J. Chem. Phys. 127, 144304 (2007).

${ }^{50}$ M. Artamonov, T.-S. Ho, and H. Rabitz, Chem. Phys. 305, 213 (2004).

${ }^{51}$ Y. Kurosaki, M. Artamonov, T.-S. Ho, and H. Rabitz, J. Chem. Phys. 131, 044306 (2009).

${ }^{52}$ J. C. Tully, J. Chem. Phys. 93, 1061 (1990).

${ }^{53}$ K. Takahashi, S. Hayashi, Y. Matsumi, N. Taniguchi, and S. Hayashida, J. Geophys. Res. 107, 4440, doi:10.1029/2001JD002048 (2002).

${ }^{54} \mathrm{~S}$. Yu. Grebenshchikov, private communication (2009).

${ }^{55}$ Z.-W. Qu, H. Zhu, M. Tashiro, R. Schinke, and S. C. Farantos, J. Chem. Phys. 120, 6811 (2004)

${ }^{56}$ S. Voigt, J. Orphal, K. Bogumil, and J. P. Burrows, J. Photochem. Photobiol. Chem. 143, 1 (2001).
${ }^{57}$ H. Zhu, Z.-W. Qu, S. Yu. Grebenshchikov, R. Schinke, J. Malicet, J. Brion, and D. Daumont, J. Chem. Phys. 122, 024310 (2005).

${ }^{58}$ A. Sinha, D. Imre, J. H. Goble, Jr., and J. L. Kinsey, J. Chem. Phys. 84, 6108 (1986).

${ }^{59}$ N. Taniguchi, S. Hayashida, K. Takahashi, and Y. Matsumi, Atmos. Chem. Phys. 3, 1293 (2003).

${ }^{60}$ I. A. Cooper, P. J. Neill, and J. R. Wiesenfeld, J. Geophys. Res. 98, 12795, doi:10.1029/93JD00763 (1993).

${ }^{61}$ R. Atkinson, D. L. Baulch, R. A. Cox, J. N. Crowley, R. F. Hampson, R. G. Hynes, M. E. Jenkin, M. J. Rossi, and J. Troe, Atmos. Chem. Phys. 4, 1461 (2004)

${ }^{62}$ N. Taniguchi, K. Takahashi, and Y. Matsumi, J. Phys. Chem. A 104, 8936 (2000)

${ }^{63}$ S. M. Ball, G. Hancock, S. E. Martin, and J. C. Pinot de Moira, Chem. Phys. Lett. 264, 531 (1997).

${ }^{64}$ R. K. Talukdar, M. K. Gilles, F. Battin-Leclerc, A. R. Ravishankara, J.-M. Fracheboud, J. J. Orlando, and G. S. Tyndall, Geophys. Res. Lett. 24, 1091, doi:10.1029/97GL00831 (1997).

${ }^{65}$ R. K. Talukdar, C. A. Langfellow, M. K. Gilles, and A. R. Ravishankara, Geophys. Res. Lett. 25, 143, doi:10.1029/97GL03354 (1998).

${ }^{66}$ M. Trolier and J. R. Wiesenfeld, J. Geophys. Res. 93, 7119, doi:10.1029/ JD093iD06p07119 (1988).

${ }^{67}$ S. Nishida, F. Taketani, K. Takahashi, and Y. Matsumi, J. Phys. Chem. A 108, 2710 (2004)

${ }^{68}$ P. Rosmus, P. Palmieri, and R. Schinke, J. Chem. Phys. 117, 4871 (2002).

${ }^{69}$ M. Tashiro and R. Schinke, J. Chem. Phys. 119, 10186 (2003).

${ }^{70}$ M. H. Palmer and A. D. Nelson, Mol. Phys. 100, 3601 (2002). 